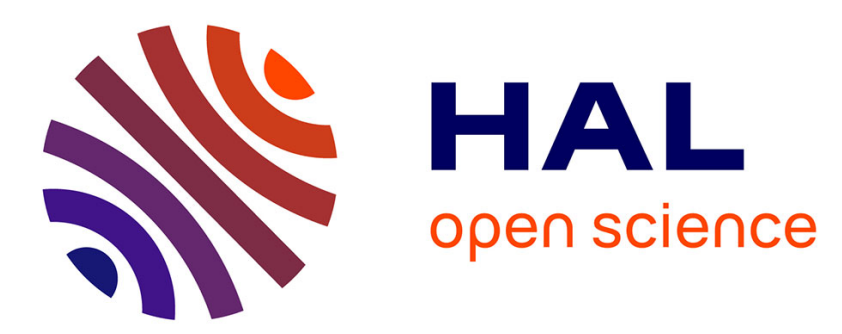

\title{
Polynomiality of Plancherel averages of hook-content summations for strict, doubled distinct and self-conjugate partitions
}

Guo-Niu Han, Huan Xiong

\section{- To cite this version:}

Guo-Niu Han, Huan Xiong. Polynomiality of Plancherel averages of hook-content summations for strict, doubled distinct and self-conjugate partitions. Journal of Combinatorial Theory, Series A, 2019, 168, pp.50-83. 10.1016/j.jcta.2019.05.012 . hal-02165514

\section{HAL Id: hal-02165514 \\ https://hal.science/hal-02165514}

Submitted on 26 Jun 2019

HAL is a multi-disciplinary open access archive for the deposit and dissemination of scientific research documents, whether they are published or not. The documents may come from teaching and research institutions in France or abroad, or from public or private research centers.
L'archive ouverte pluridisciplinaire HAL, est destinée au dépôt et à la diffusion de documents scientifiques de niveau recherche, publiés ou non, émanant des établissements d'enseignement et de recherche français ou étrangers, des laboratoires publics ou privés. 


\title{
Polynomiality of Plancherel averages of hook-content summations for strict, doubled distinct and self-conjugate partitions
}

\author{
Guo-Niu Han and Huan Xiong*
}

\begin{abstract}
The polynomiality of shifted Plancherel averages for summations of contents of strict partitions were established by the authors and Matsumoto independently in 2015, which is the key to determining the limit shape of random shifted Young diagram, as explained by Matsumoto. In this paper, we prove the polynomiality of shifted Plancherel averages for summations of hook lengths of strict partitions, which is an analog of the authors and Matsumoto's results on contents of strict partitions.

In 2009, the first author proved the Nekrasov-Okounkov formula on hook lengths for integer partitions by using an identity of Macdonald in the framework of type $\widetilde{A}$ affine root systems, and conjectured that the Plancherel averages of some summations over the set of all partitions of size $n$ are always polynomials in $n$. This conjecture was generalized and proved by Stanley. Recently, Pétréolle derived two Nekrasov-Okounkov type formulas for $\widetilde{C}$ and $\widetilde{C}^{\sim}$ which involve doubled distinct and self-conjugate partitions. Inspired by all those previous works, we establish the polynomiality of $t$-Plancherel averages of some hook-content summations for doubled distinct and self-conjugate partitions.
\end{abstract}

\section{INTRODUCTION}

A strict partition is a finite strict decreasing sequence of positive integers $\bar{\lambda}=$ $\left(\bar{\lambda}_{1}, \bar{\lambda}_{2}, \ldots, \bar{\lambda}_{\ell}\right)$ (see $\left.[17,31]\right)$. The integer $|\bar{\lambda}|=\sum_{1<i<\ell} \bar{\lambda}_{i}$ is called the size and $\ell(\bar{\lambda})=\ell$ is called the length of $\bar{\lambda}$. For convenience, let $\bar{\lambda}_{i}=0$ for $i>\ell(\bar{\lambda})$. A strict partition $\bar{\lambda}$ is identified with its shifted Young diagram, which means that the $i$-th row of the usual Young diagram is shifted to the right by $i$ boxes. A standard shifted Young tableau of the shape $\lambda$ is obtained by filling in the boxes of the shifted Young diagram of $\lambda$ with numbers from 1 to $|\lambda|$ such that the numbers strictly increase along every row and every column. The shifted Plancherel measure $\mathbb{P}_{n}$ for strict partitions with size $n$ is defined by (see $[3,13,18,19,20]$ )

$$
\mathbb{P}_{n}(\bar{\lambda}):=\frac{2^{n-\ell(\bar{\lambda})}\left(f_{\bar{\lambda}}\right)^{2}}{n !}=\frac{2^{n-\ell(\bar{\lambda})} f_{\bar{\lambda}}}{H(\bar{\lambda})}
$$

where $f_{\bar{\lambda}}$ is the number of standard shifted Young tableaux of shape $\bar{\lambda}$, and $H(\bar{\lambda})$ is the product of all hook lengths of boxes in the shifted Young diagram of $\bar{\lambda}$. For a function $\varphi$ on the set of strict partitions, its shifted Plancherel average is defined

2010 Mathematics Subject Classification. 05A15, 05A17, 05A19, 05E05, 05E10, 11P81.

Key words and phrases. random partition, Plancherel measure, strict partition, doubled distinct partition, self-conjugate partition, hook length, content, difference operator.

* Huan Xiong is the corresponding author. 
by (see [18])

$$
\mathbb{E}_{n}[\varphi]:=\sum_{|\bar{\lambda}|=n} \mathbb{P}_{n}(\bar{\lambda}) \varphi(\bar{\lambda})=\sum_{|\bar{\lambda}|=n} \frac{2^{n-\ell(\bar{\lambda})}\left(f_{\bar{\lambda}}\right)^{2}}{n !} \varphi(\bar{\lambda}) .
$$

The polynomiality of shifted Plancherel averages for functions related to contents of strict partitions (the definition for contents and hook lengths of strict partitions are given in the beginning of Section 2) were established by the authors [12] and Matsumoto [18] recently.

Theorem $1.1([12,18])$. Suppose that $Q$ is a given symmetric function. Then

$$
\mathbb{E}_{n}\left[Q\left(\left(\begin{array}{c}
c_{\square} \\
2
\end{array}\right): \square \in \bar{\lambda}\right)\right]=\sum_{|\bar{\lambda}|=n} \frac{2^{n-\ell(\bar{\lambda})} f_{\bar{\lambda}}}{H(\bar{\lambda})} Q\left(\left(\begin{array}{c}
c_{\square} \\
2
\end{array}\right): \square \in \bar{\lambda}\right)
$$

is a polynomial in $n$, where $c_{\square}$ is the content of the box $\square$ in the shifted Young diagram of $\bar{\lambda}$.

Theorem 1.1 is important in the study of the limit shape problems of random shifted Young diagram, as explained by Matsumoto [19]. In Section 2 we derive the following hook length analog for Theorem 1.1.

Theorem 1.2. Suppose that $k$ is a given nonnegative integer and $\bar{\mu}$ is a given strict partition. Then,

$$
\sum_{|\bar{\lambda} / \bar{\mu}|=n} \frac{2^{|\bar{\lambda}|-\ell(\bar{\lambda})} f_{\bar{\lambda} / \bar{\mu}}}{H(\bar{\lambda})}\left(2 \sum_{h \in \mathcal{H}(\bar{\lambda})} h^{2 k}+\left(4^{k}-1\right) \sum_{i=1}^{\ell(\bar{\lambda})} \bar{\lambda}_{i}^{2 k}\right)
$$

is a polynomial in $n$ of degree at most $k+1$, where $f_{\bar{\lambda} / \bar{\mu}}$ is the number of standard skew shifted Young tableaux of shape $\bar{\lambda} / \bar{\mu}$, and $\mathcal{H}(\bar{\lambda})$ is the multiset of hook lengths of boxes in the shifted Young diagram of $\bar{\lambda}$.

We mention that the term $\left(4^{k}-1\right) \sum_{i=1}^{\ell(\bar{\lambda})} \bar{\lambda}_{i}^{2 k}$ is necessary in the formula (1.1), since $\mathbb{E}_{n}\left[\sum_{h \in \mathcal{H}(\bar{\lambda})} h^{2 k}\right]$ is not a polynomial of $n$ when $k \geq 1$.

The following so-called Nekrasov-Okounkov formula

$$
\sum_{\lambda \in \mathcal{P}} q^{|\lambda|} \prod_{h \in \mathcal{H}(\lambda)}\left(1-\frac{z}{h^{2}}\right)=\prod_{k \geq 1}\left(1-q^{k}\right)^{z-1},
$$

where $\mathcal{P}$ is the set of all integer partitions $\lambda$ with $|\lambda|$ denoting the size of $\lambda$ and $\mathcal{H}(\lambda)$ the multiset of hook lengths associated with $\lambda$ (see [7]), was discovered independently several times: first, by Nekrasov and Okounkov in their study of the theory of Seiberg-Witten on supersymmetric gauges in particle physics [21]; then, proved by Westbury using D'Arcais polynomials [33]; finally, by the first author using an identity of Macdonald [17] in the framework of type $\widetilde{A}$ affine root systems [7]. Moreover, he asked to find Nekrasov-Okounkov type formulas associated with other root systems [8, Problem 6.4], and conjectured that the Plancherel average

$$
n ! \sum_{|\lambda|=n} \frac{1}{H(\lambda)^{2}} \sum_{h \in \mathcal{H}(\lambda)} h^{2 k}
$$



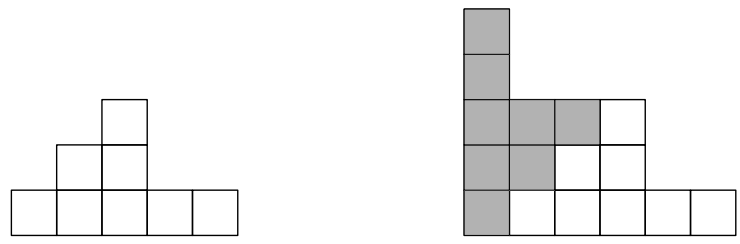

FiguRE 1. From strict partitions to doubled distinct partitions.

is always a polynomial in $n$ for any $k \in \mathbb{N}$, where $H(\lambda)=\prod_{h \in \mathcal{H}(\lambda)} h$. This conjecture was proved by Stanley in a more general form. In particular, he showed that

$$
n ! \sum_{|\lambda|=n} \frac{1}{H(\lambda)^{2}} Q_{1}\left(h^{2}: h \in \mathcal{H}(\lambda)\right) Q_{2}(c: c \in \mathcal{C}(\lambda))
$$

is a polynomial in $n$ for any symmetric functions $Q_{1}$ and $Q_{2}$, where $\mathcal{C}(\lambda)$ is the multiset of contents associated with $\lambda$ (see [29]). For some special functions $Q_{1}$ and $Q_{2}$ the latter polynomial has explicit expression, as shown by Fujii-KannoMoriyama-Okada [5] and Panova [24].

The doubled distinct partition of a strict partition $\bar{\lambda}$, denoted by $\bar{\lambda} \bar{\lambda}$, is defined to be the usual partition whose Young diagram is obtained by adding $\bar{\lambda}_{i}$ boxes to the $i$-th column of the shifted Young diagram of $\bar{\lambda}$ for $1 \leq i \leq \ell(\bar{\lambda})($ see $[6,25,26])$. For example, $(6,4,4,1,1)$ is the doubled distinct partition of $(5,2,1)$ (see Figure 1).

For each usual partition $\lambda$, let $\lambda^{\prime}$ denote the conjugate partition of $\lambda$ (see [6, $17,25,26]$ ). A usual partition $\lambda$ is called self-conjugate if $\lambda=\lambda^{\prime}$. The set of all doubled distinct partitions and the set of all self-conjugate partitions are denoted by $\mathcal{D D}$ and $\mathcal{S C}$ respectively. For each positive integer $t$, let

$$
\mathcal{H}_{t}(\lambda)=\{h \in \mathcal{H}(\lambda): h \equiv 0 \quad(\bmod t)\}
$$

be the multiset of hook lengths of multiples of $t$. Write $H_{t}(\lambda)=\prod_{h \in \mathcal{H}_{t}(\lambda)} h$.

Recently, Pétréolle derived two Nekrasov-Okounkov type formulas for $\widetilde{C}$ and $\widetilde{C}^{\sim}$ which involve doubled distinct and self-conjugate partitions. In particular, he obtained the following two formulas in $[25,26]$.

Theorem 1.3 (Pétréolle $[25,26]$ ). For positive integers $n$ and $t$ we have

$$
\begin{aligned}
& \sum_{\substack{\lambda \in \mathcal{D} \mathcal{D},|\lambda|=2 n t \\
\# \mathcal{H}_{t}(\lambda)=2 n}} \frac{1}{H_{t}(\lambda)}=\frac{1}{(2 t)^{n} n !}, \quad \text { if } t \text { is odd; } \\
& \sum_{\substack{\lambda \in \mathcal{S} \mathcal{C},|\lambda|=2 n t \\
\# \mathcal{H}_{t}(\lambda)=2 n}} \frac{1}{H_{t}(\lambda)}=\frac{1}{(2 t)^{n} n !}, \quad \text { if } t \text { is even. }
\end{aligned}
$$

We call

$$
\mathbb{P}_{t, n}^{\mathcal{D D}}(\lambda):=\frac{(2 t)^{n} n !}{H_{t}(\lambda)} \quad(\mathrm{t} \text { odd })
$$

and

$$
\mathbb{P}_{t, n}^{\mathcal{S C}}(\lambda):=\frac{(2 t)^{n} n !}{H_{t}(\lambda)} \quad(\mathrm{t} \text { even })
$$


the $t$-Plancherel measure for doubled distinct and self-conjugate partitions with size $n$, respectively. For a function $\varphi$ on the sets of doubled distinct or self-conjugate partitions, its $t$-Plancherel average is defined by

$$
\mathbb{E}_{t, n}^{\mathcal{D D}}[\varphi]:=\sum_{\substack{\lambda \in \mathcal{D} \mathcal{D},|\lambda|=2 n t \\
\# \mathcal{H}_{t}(\lambda)=2 n}} \mathbb{P}_{t, n}^{\mathcal{D D}} \varphi(\lambda)=\sum_{\begin{array}{c}
\lambda \in \mathcal{D} \mathcal{D},|\lambda|=2 n t \\
\# \mathcal{H}_{t}(\lambda)=2 n
\end{array}} \frac{(2 t)^{n} n !}{H_{t}(\lambda)} \varphi(\lambda) \quad(\mathrm{t} \text { odd })
$$

and

$$
\left.\mathbb{E}_{t, n}^{\mathcal{S C}}[\varphi]:=\sum_{\substack{\lambda \in \mathcal{S C},|\lambda|=2 n t \\
\# \mathcal{H}_{t}(\lambda)=2 n}} \mathbb{P}_{t, n}^{\mathcal{S C}} \varphi(\lambda)=\sum_{\begin{array}{c}
\lambda \in \mathcal{S C},|\lambda|=2 n t \\
\# \mathcal{H}_{t}(\lambda)=2 n
\end{array}} \frac{(2 t)^{n} n !}{H_{t}(\lambda)} \varphi(\lambda) \quad \text { (t even }\right)
$$

respectively.

Actually, the $t$-Plancherel measure in this paper can be generalized to be defined on the set of general partitions (see [4]). As we know, the linear and projective representations of symmetric groups can be labelled bijectively by partitions and strict partitions, respectively (see [17]). Therefore, the ordinary and shifted Plancherel measure gives natural probability measure on such representations. Similarly, when $t$ is a prime number, the $t$-blocks in the modular representations of symmetric groups can be labelled bijectively by $t$-core partitions. In fact, the famous Nakayama conjecture [14, Theorem 6.2.21] states that two irreducible characters of the symmetric groups are in the same $t$-block if and only if their corresponding partitions have the same $t$-core. The $t$-Plancherel measure thus gives natural probability measure on $t$-blocks in the modular representations of symmetric groups. Also, as the ordinary and shifted Plancherel measure are closely related to certain numbers of paths in Young's lattice, our $t$-Plancherel measure can be seen as a generalized probability measure arising from counting the paths (see (4.3) and (5.2)) in Stanley's $t$-differential poset theory [30].

Inspired by previous works, we establish the polynomiality of $t$-Plancherel averages of some hook-content summations for doubled distinct and self-conjugate partitions. Our main result is stated next.

Theorem 1.4. For any given symmetric functions $Q_{1}$ and $Q_{2}$, the following two $t$-Plancherel averages

$$
\mathbb{E}_{t, n}^{\mathcal{D D}}\left[Q_{1}\left(h^{2}: h \in \mathcal{H}(\lambda)\right) Q_{2}(c: c \in \mathcal{C}(\lambda))\right] \quad(t \quad \text { odd })
$$

and

$$
\mathbb{E}_{t, n}^{\mathcal{S C}}\left[Q_{1}\left(h^{2}: h \in \mathcal{H}(\lambda)\right) Q_{2}(c: c \in \mathcal{C}(\lambda))\right] \quad(t \text { even })
$$

are polynomials in $n$ and $t$.

In fact, the degrees of the two polynomials in $n$ and $t$ can be estimated explicitly in terms of $Q_{1}$ and $Q_{2}$ (see Corollary 4.8 and Theorem 5.4). When $Q_{1}$ and $Q_{2}$ are two constant symmetric functions, we derive Theorem 1.3. Other specializations are listed as follows.

Corollary 1.5. We have

$$
(2 t)^{n} n ! \sum_{\substack{\lambda \in \mathcal{D} \mathcal{D},|\lambda|=2 n t \\ \# \mathcal{H}_{t}(\lambda)=2 n}} \frac{1}{H_{t}(\lambda)} \sum_{h \in \mathcal{H}(\lambda)} h^{2}=6 t^{2} n^{2}+\frac{1}{3}\left(t^{2}-6 t+2\right) t n \quad(t \text { odd }),
$$




$$
\begin{aligned}
&(2 t)^{n} n ! \sum_{\substack{\lambda \in \mathcal{S},|\lambda|=2 n t \\
\# \mathcal{H}_{t}(\lambda)=2 n}} \frac{1}{H_{t}(\lambda)} \sum_{h \in \mathcal{H}(\lambda)} h^{2}=6 t^{2} n^{2}+\frac{1}{3}\left(t^{2}-6 t-1\right) t n \quad(t \text { even }), \\
&(2 t)^{n} n ! \sum_{\substack{\lambda \in \mathcal{D} \mathcal{D},|\lambda|=2 n t \\
\# \mathcal{H}_{t}(\lambda)=2 n}} \frac{1}{H_{t}(\lambda)} \sum_{c \in \mathcal{C}(\lambda)} c^{2}=2 t^{2} n^{2}+\frac{1}{3}\left(t^{2}-6 t+2\right) t n \quad(t \text { odd }), \\
&(2 t)^{n} n ! \sum_{\substack{\lambda \in \mathcal{S C},|\lambda|=2 n t \\
\# \mathcal{H}_{t}(\lambda)=2 n}} \frac{1}{H_{t}(\lambda)} \sum_{c \in \mathcal{C}(\lambda)} c^{2}=2 t^{2} n^{2}+\frac{1}{3}\left(t^{2}-6 t-1\right) t n \quad(t \text { even }) .
\end{aligned}
$$

The rest of the paper is essentially devoted to completing the proof of Theorems 1.2 and 1.4. In Section 2 we study the difference operator for strict partitions and prove Theorem 1.2. The polynomiality of summations in (1.4) for $t=1$ with $Q_{1}=1$ or $Q_{2}=1$ has an equivalent statement in terms of strict partitions, whose proof is also given in Section 2. After recalling some basic definitions and properties of Littlewood decomposition in Section 3, the doubled distinct and self-conjugate cases of Theorem 1.4 are proved in Sections 4 and 5 respectively. Finally, Corollary 1.5 is proved in Section 6. The basic idea in the proofs of Theorems 1.2 and 1.4 is to translate the problem on Plancherel averages to the calculation of some higherorder difference operators on partitions and strict partitions (see Theorems 2.5, 4.3 and 5.3). Then the polynomiality of Plancherel averages corresponds to the vanishing of such higher-order difference operators on specific functions of hook lengths and contents. Therefore, we establish the relation between higher-order and lower order difference operators by studying the combinatorial properties of hook lengths, contents, inner and outer corners under the Littlewoord decomposition, which then be used to inductively prove the vanishing properties of difference operators (see Theorems 2.6, 4.7 and 5.4).

\section{Polynomiality for Strict and Doubled Distinct Partitions}

In this section we prove Theorem 1.2 and an equivalent statement of the polynomiality of (1.4) for $t=1$ with $Q_{1}=1$ or $Q_{2}=1$. Let $\bar{\lambda}=\left(\bar{\lambda}_{1}, \bar{\lambda}_{2}, \ldots, \bar{\lambda}_{\ell}\right)$ be a strict partition. The leftmost box in the $i$-th row of the shifted Young diagram of $\bar{\lambda}$ has coordinate $(i, i+1)$. The hook length of the $(i, j)$-box, denoted by $h_{(i, j)}$, is the number of boxes exactly to the right, or exactly above, or the box itself, plus $\bar{\lambda}_{j}$. For example, consider the box $\square=(i, j)=(1,3)$ in the shifted Young diagram of the strict partition $(7,5,4,1)$. There are 1 and 5 boxes below and to the right of the box $\square$ respectively. Since $\bar{\lambda}_{3}=4$, the hook length of $\square$ is equal to $1+5+1+4=11$, as illustrated in Figure 2. The content of $\square=(i, j)$ is defined to be $c_{\square}=j-i$, so that the leftmost box in each row has content 1 . Also, let $\mathcal{H}(\bar{\lambda})$ be the multiset of hook lengths of boxes. The hook length and content multisets of the doubled distinct partition $\bar{\lambda} \bar{\lambda}$ can be obtained from $\mathcal{H}(\bar{\lambda})$ and $\mathcal{C}(\bar{\lambda})$ by the following relations:

$$
\begin{aligned}
\mathcal{H}(\bar{\lambda} \bar{\lambda}) & =\mathcal{H}(\bar{\lambda}) \cup \mathcal{H}(\bar{\lambda}) \cup\left\{2 \bar{\lambda}_{1}, 2 \bar{\lambda}_{2}, \ldots, 2 \bar{\lambda}_{\ell}\right\} \backslash\left\{\bar{\lambda}_{1}, \bar{\lambda}_{2}, \ldots, \bar{\lambda}_{\ell}\right\} \\
\mathcal{C}(\bar{\lambda} \bar{\lambda}) & =\mathcal{C}(\bar{\lambda}) \cup\{1-c \mid c \in \mathcal{C}(\bar{\lambda})\} .
\end{aligned}
$$

The proofs of (2.1) and (2.2) can be derived by splitting the Young diagram of the partition $\bar{\lambda} \bar{\lambda}$ into two sets $S_{1}(\bar{\lambda} \bar{\lambda}):=\{(i, j) \in \bar{\lambda} \bar{\lambda}: i<j\}$ and $S_{2}(\bar{\lambda} \bar{\lambda}):=\{(i, j) \in$ $\bar{\lambda} \bar{\lambda}: i \geq j\}$. By definition, the hook length and content multisets for $S_{1}(\bar{\lambda} \bar{\lambda})$ are 

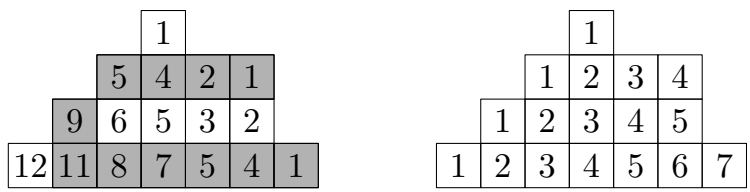

Figure 2. The shifted Young diagram, the hook lengths and the contents of the strict partition $(7,5,4,1)$.

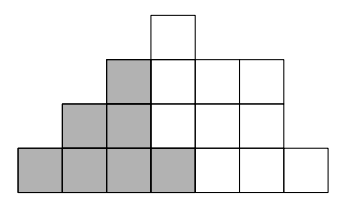

Figure 3. The skew shifted Young diagram of the skew strict partition $(7,5,4,1) /(4,2,1)$.

$\mathcal{H}(\bar{\lambda})$ and $\mathcal{C}(\bar{\lambda})$ respectively, while the hook length and content multisets for $S_{2}(\bar{\lambda} \bar{\lambda})$ are $\mathcal{H}(\bar{\lambda}) \cup\left\{2 \bar{\lambda}_{1}, 2 \bar{\lambda}_{2}, \ldots, 2 \bar{\lambda}_{\ell}\right\} \backslash\left\{\bar{\lambda}_{1}, \bar{\lambda}_{2}, \ldots, \bar{\lambda}_{\ell}\right\}$ and $\{1-c \mid c \in \mathcal{C}(\bar{\lambda})\}$, respectively (for a detailed explanation, see $[25$, p. 9$]$ ).

For two strict partitions $\bar{\lambda}$ and $\bar{\mu}$, we write $\bar{\lambda} \supseteq \bar{\mu}$ if $\bar{\lambda}_{i} \geq \bar{\mu}_{i}$ for any $i \geq 1$. In this case, the skew strict partition $\bar{\lambda} / \bar{\mu}$ is identical with the skew shifted Young diagram. For example, the skew strict partition $(7,5,4,1) /(4,2,1)$ is represented by the white boxes in Figure 3 . Recall that $f_{\bar{\lambda}}$ (resp. $f_{\bar{\lambda} / \bar{\mu}}$ ) denotes the number of standard shifted Young tableaux of shape $\bar{\lambda}(\operatorname{resp} . \bar{\lambda} / \bar{\mu})$. The following formulas for strict partitions are well-known (see $[2,28,32])$ :

$$
f_{\bar{\lambda}}=\frac{|\bar{\lambda}| !}{H(\bar{\lambda})} \quad \text { and } \quad \frac{1}{n !} \sum_{|\bar{\lambda}|=n} 2^{n-\ell(\bar{\lambda})} f_{\bar{\lambda}}^{2}=1 .
$$

Identity (1.2) with $t=1$, obtained by Pétréolle, becomes

$$
\sum_{\lambda \in \mathcal{D} \mathcal{D},|\lambda|=2 n} \frac{1}{H(\lambda)}=\frac{1}{2^{n} n !},
$$

which is equivalent to the second identity of (2.3) in view of (2.1).

For a strict partition $\bar{\lambda}$, the outer corners (see [12]) are the boxes which can be removed in such a way that after removal the resulting diagram is still a shifted Young diagram of a strict partition. The coordinates of outer corners are denoted by $\left(\alpha_{1}, \beta_{1}\right), \ldots,\left(\alpha_{m}, \beta_{m}\right)$ such that $\alpha_{1}>\alpha_{2}>\cdots>\alpha_{m}$. Let $y_{j}:=\beta_{j}-\alpha_{j}$ $(1 \leq j \leq m)$ be the contents of outer corners. We set $\alpha_{m+1}=0, \beta_{0}=\ell(\bar{\lambda})+1$ and call $\left(\alpha_{1}, \beta_{0}\right),\left(\alpha_{2}, \beta_{1}\right), \ldots,\left(\alpha_{m+1}, \beta_{m}\right)$ the inner corners of $\bar{\lambda}$. Let $x_{i}=\beta_{i}-\alpha_{i+1}$ be the contents of inner corners for $0 \leq i \leq m$ (see Figure 4 ). The following relation of $x_{i}$ and $y_{j}$ are obvious:

$$
x_{0}=1 \leq y_{1}<x_{1}<y_{2}<x_{2}<\cdots<y_{m}<x_{m} .
$$

Notice that $x_{0}=y_{1}=1$ iff $\bar{\lambda}_{\ell(\bar{\lambda})}=1$. Let $\bar{\lambda}^{i+}=\bar{\lambda} \bigcup\left\{\square_{i}\right\}$ such that $c_{\square_{i}}=$ $x_{i}$ for $0 \leq i \leq m$. Therefore, $\bar{\lambda}^{i+}$ is the partition obtained by adding the box $\square_{i}=\left(\alpha_{i+1}+1, \beta_{i}+1\right)$ to $\lambda$. Here $\bar{\lambda}^{0+}$ does not exist if $y_{1}=1$. The set of 


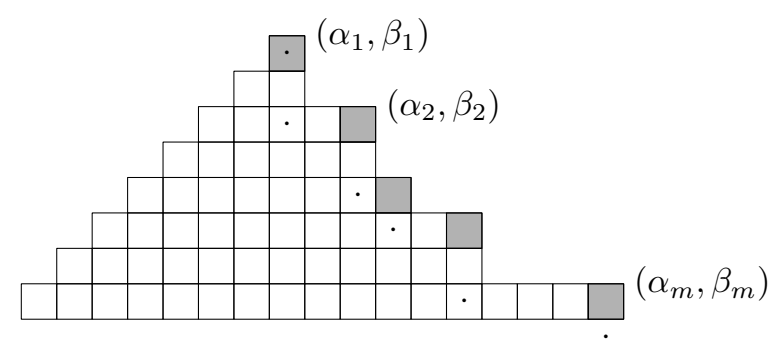

Figure 4. A strict partition and its corners. The outer corners are labelled with $\left(\alpha_{i}, \beta_{i}\right)(i=1,2, \ldots, m)$. The inner corners are indicated by the dot symbol ".".

contents of inner corners and the set of contents of outer corners of $\bar{\lambda}$ are denoted by $X(\bar{\lambda})=\left\{x_{0}, x_{1}, \ldots, x_{m}\right\}$ and $Y(\bar{\lambda})=\left\{y_{1}, y_{2}, \ldots, y_{m}\right\}$ respectively. The following relations between the hook lengths of $\bar{\lambda}$ and $\bar{\lambda}^{i+}$ are established in [12].

Theorem 2.1 (Theorem 3.1 of [12]). Let $\bar{\lambda}$ be a strict partition with $X(\bar{\lambda})=$ $\left\{x_{0}, x_{1}, \ldots, x_{m}\right\}$ and $Y(\bar{\lambda})=\left\{y_{1}, y_{2}, \ldots, y_{m}\right\}$. For $1 \leq i \leq m$, we have

$$
\begin{gathered}
\mathcal{H}(\bar{\lambda}) \cup\left\{1, x_{i}, 2 x_{i}-2\right\} \cup\left\{\left|x_{i}-x_{j}\right|: 1 \leq j \leq m, j \neq i\right\} \\
\cup\left\{x_{i}+x_{j}-1: 1 \leq j \leq m, j \neq i\right\} \\
=\mathcal{H}\left(\bar{\lambda}^{i+}\right) \cup\left\{\left|x_{i}-y_{j}\right|: 1 \leq j \leq m\right\} \cup\left\{x_{i}+y_{j}-1: 1 \leq j \leq m\right\}
\end{gathered}
$$

and

$$
\frac{H(\bar{\lambda})}{H\left(\bar{\lambda}^{i+}\right)}=\frac{1}{2} \cdot \frac{\prod_{\substack{1 \leq j \leq m \\
0 \leq j \leq m \\
j \neq i}}\left(\left(\begin{array}{c}
x_{i} \\
2
\end{array}\right)-\left(\begin{array}{c}
y_{j} \\
2
\end{array}\right)\right)}{\left.\left.\prod_{i}\right)-\left(\begin{array}{c}
x_{j} \\
2
\end{array}\right)\right)} .
$$

If $y_{1}>1$, we have

$$
\begin{aligned}
& \mathcal{H}(\bar{\lambda}) \cup\left\{1, x_{1}, x_{1}-1, x_{2}, x_{2}-1, \cdots, x_{m}, x_{m}-1\right\} \\
= & \mathcal{H}\left(\bar{\lambda}^{0+}\right) \cup\left\{y_{1}, y_{1}-1, y_{2}, y_{2}-1, \cdots, y_{m}, y_{m}-1\right\}
\end{aligned}
$$

and

$$
\frac{H(\bar{\lambda})}{H\left(\bar{\lambda}^{0+}\right)}=\frac{\prod_{1 \leq j \leq m}\left(\left(\begin{array}{c}
x_{0} \\
2
\end{array}\right)-\left(\begin{array}{c}
y_{j} \\
2
\end{array}\right)\right)}{\prod_{1 \leq j \leq m}\left(\left(\begin{array}{c}
x_{0} \\
2
\end{array}\right)-\left(\begin{array}{c}
x_{j} \\
2
\end{array}\right)\right)} .
$$

Example 2.1. Let $\bar{\lambda}=(5,4,2)$. Then $X(\bar{\lambda})=\{1,3,6\}$ and $Y(\bar{\lambda})=\{2,4\}$. Also, we have $\mathcal{H}(\bar{\lambda})=\{1,1,2,2,3,4,4,5,6,7,9\}, \mathcal{H}\left(\bar{\lambda}^{1+}\right)=\{1,2,2,3,3,3,4,4,5,7,8,9\}$ and $\mathcal{H}\left(\bar{\lambda}^{0+}\right)=\{1,1,2,2,3,4,5,5,6,6,7,9\}$. Therefore it is easy to check that the following two equalities in Theorem 2.1 hold:

$$
\begin{aligned}
& \mathcal{H}(\bar{\lambda}) \cup\left\{1, x_{1}, 2 x_{1}-2\right\} \cup\left\{\left|x_{1}-x_{j}\right|: 1 \leq j \leq m, j \neq 1\right\} \\
& \cup\left\{x_{1}+x_{j}-1: 1 \leq j \leq m, j \neq 1\right\} \\
&=\mathcal{H}\left(\bar{\lambda}^{1+}\right) \cup\left\{\left|x_{1}-y_{j}\right|: 1 \leq j \leq m\right\} \cup\left\{x_{1}+y_{j}-1: 1 \leq j \leq m\right\} \\
&=\{1,1,1,2,2,3,3,3,4,4,4,5,6,7,8,9\},
\end{aligned}
$$


and

$$
\begin{aligned}
& \mathcal{H}(\bar{\lambda}) \cup\left\{1, x_{1}, x_{1}-1, x_{2}, x_{2}-1, \cdots, x_{m}, x_{m}-1\right\} \\
= & \mathcal{H}\left(\bar{\lambda}^{0+}\right) \cup\left\{y_{1}, y_{1}-1, y_{2}, y_{2}-1, \cdots, y_{m}, y_{m}-1\right\} \\
= & \{1,1,1,2,2,2,3,3,4,4,5,5,6,6,7,9\} .
\end{aligned}
$$

Let $k$ be a nonnegative integer, and $\nu=\left(\nu_{1}, \nu_{2}, \ldots, \nu_{\ell(\nu)}\right)$ be a usual partition. For arbitrary two finite alphabets $A$ and $B$, the power difference of the alphabet $A-B$ is defined by $[16, \mathrm{p} .5]$

$$
\begin{aligned}
\Psi^{k}(A, B) & :=\sum_{a \in A} a^{k}-\sum_{b \in B} b^{k}, \\
\Psi^{\nu}(A, B) & :=\prod_{j=1}^{\ell(\nu)} \Psi^{\nu_{j}}(A, B) .
\end{aligned}
$$

Let $\bar{\lambda}$ be a strict partition. We define

$$
\Phi^{\nu}(\bar{\lambda}):=\Psi^{\nu}\left(\left\{\left(\begin{array}{c}
x_{i} \\
2
\end{array}\right)\right\},\left\{\left(\begin{array}{c}
y_{i} \\
2
\end{array}\right)\right\}\right) .
$$

Theorem 2.2 (Theorem 3.5 of [12]). Let $k$ be a given nonnegative integer. Then, there exist some $\xi_{j} \in \mathbb{Q}$ such that

$$
\Phi^{k}\left(\bar{\lambda}^{i+}\right)-\Phi^{k}(\bar{\lambda})=\sum_{j=0}^{k-1} \xi_{j}\left(\begin{array}{c}
x_{i} \\
2
\end{array}\right)^{j}
$$

for every strict partition $\bar{\lambda}$ and $0 \leq i \leq m$, where $x_{0}, x_{1}, \ldots, x_{m}$ are the contents of inner corners of $\bar{\lambda}$.

We also need the following lemmas.

Lemma 2.3. Let $k$ be a given nonnegative integer. Then, there exist some constants $a_{k i j}$ such that

$$
(x-y)^{2 k}+(x+y-1)^{2 k}=\sum_{i+j \leq k} a_{k i j}\left(\begin{array}{l}
x \\
2
\end{array}\right)^{i}\left(\begin{array}{l}
y \\
2
\end{array}\right)^{j}
$$

for every $x, y \in \mathbb{C}$.

Proof. First, it is easy to check that

$$
(x-y)^{2}+(x+y-1)^{2}=4\left(\begin{array}{l}
x \\
2
\end{array}\right)+4\left(\begin{array}{l}
y \\
2
\end{array}\right)+1
$$

and

$$
(x-y)^{2}(x+y-1)^{2}=\left(2\left(\begin{array}{l}
x \\
2
\end{array}\right)-2\left(\begin{array}{l}
y \\
2
\end{array}\right)\right)^{2},
$$

which imply that the claim holds for $k=1$. Let $A_{k}(x, y)=(x-y)^{2 k}+(x+y-1)^{2 k}$. For $k \geq 2$, we have

$$
A_{k}(x, y)=A_{1}(x, y) A_{k-1}(x, y)-(x-y)^{2}(x+y-1)^{2} A_{k-2}(x, y) .
$$

Then by induction the lemma holds for $k \geq 1$. 
Lemma 2.4 (Theorem 3.2 of [12]). Let $k$ be a nonnegative integer. Then, there exist some $\xi_{\nu} \in \mathbb{Q}$ indexed by usual partitions $\nu$ such that

$$
\sum_{0 \leq i \leq m} \frac{\prod_{\substack { 1 \leq j \leq m \\
\begin{subarray}{c}{0 \leq j \leq m \\
j \neq i{ 1 \leq j \leq m \\
\begin{subarray} { c } { 0 \leq j \leq m \\
j \neq i } }\end{subarray}}\left(a_{i}-b_{j}\right)}{\left.\prod_{i}-a_{j}\right)} a_{i}^{k}=\sum_{|\nu| \leq k} \xi_{\nu} \Psi^{\nu}\left(\left\{a_{i}\right\},\left\{b_{i}\right\}\right)
$$

for arbitrary complex numbers $a_{0}<a_{1}<\cdots<a_{m}$ and $b_{1}<b_{2}<\cdots<b_{m}$.

We define the difference operator $\bar{D}$ for strict partitions by

$$
\bar{D}(g(\bar{\lambda})):=2 \sum_{i=1}^{m} g\left(\bar{\lambda}^{i+}\right)+g\left(\bar{\lambda}^{0+}\right)-g(\bar{\lambda})
$$

where $\bar{\lambda}$ is a strict partition and $g$ is a function of strict partitions. In the above definition, the symbol $g\left(\bar{\lambda}^{0+}\right)$ takes the value 0 if $\bar{\lambda}^{0+}$ does not exist, or equivalently if $\bar{\lambda}_{\ell(\bar{\lambda})}=1$. By Theorem 2.1, we have

$$
\bar{D}\left(\frac{1}{H(\bar{\lambda})}\right)=0
$$

Theorem 2.5 (Theorem 2.3 of [12]). Let $g$ be a function of strict partitions and $\bar{\mu}$ be a given strict partition. Then we have

$$
\sum_{|\bar{\lambda} / \bar{\mu}|=n} 2^{|\bar{\lambda}|-|\bar{\mu}|-\ell(\bar{\lambda})+\ell(\bar{\mu})} f_{\bar{\lambda} / \bar{\mu}} g(\bar{\lambda})=\sum_{k=0}^{n}\left(\begin{array}{l}
n \\
k
\end{array}\right) \bar{D}^{k} g(\bar{\mu})
$$

and

$$
\bar{D}^{n} g(\bar{\mu})=\sum_{k=0}^{n}(-1)^{n+k}\left(\begin{array}{l}
n \\
k
\end{array}\right) \sum_{|\bar{\lambda} / \bar{\mu}|=k} 2^{|\bar{\lambda}|-|\bar{\mu}|-\ell(\bar{\lambda})+\ell(\bar{\mu})} f_{\bar{\lambda} / \bar{\mu}} g(\bar{\lambda}) .
$$

In particular, if there exists some positive integer $r$ such that $\bar{D}^{r} g(\bar{\lambda})=0$ for every strict partition $\bar{\lambda}$, then the left-hand side of (2.10) is a polynomial of $n$ with degree at most $r-1$.

For each usual partition $\delta$ let

$$
p^{\delta}(\bar{\lambda}):=\Psi^{\delta}\left(\left\{h^{2}: h \in \mathcal{H}(\bar{\lambda} \bar{\lambda})\right\}, \emptyset\right) .
$$

By (2.1), we have

$$
p^{k}(\bar{\lambda})=\sum_{h \in \mathcal{H}(\bar{\lambda} \bar{\lambda})} h^{2 k}=2 \sum_{h \in \mathcal{H}(\bar{\lambda})} h^{2 k}+\left(4^{k}-1\right) \sum_{i=1}^{\ell(\bar{\lambda})} \bar{\lambda}_{i}^{2 k}
$$

for a nonnegative integer $k$.

We will prove the following result, which implies Theorem 1.2.

Theorem 2.6. Suppose that $\nu$ and $\delta$ are two given usual partitions. Then,

$$
\bar{D}^{r}\left(\frac{p^{\delta}(\bar{\lambda}) \Phi^{\nu}(\bar{\lambda})}{H(\bar{\lambda})}\right)=0
$$


for every strict partition $\bar{\lambda}$, where $r=|\delta|+\ell(\delta)+|\nu|+1$. Consequently, for a given strict partition $\bar{\mu}$,

$$
\sum_{|\bar{\lambda} / \bar{\mu}|=n} \frac{2^{|\bar{\lambda}|-\ell(\bar{\lambda})} f_{\bar{\lambda} / \bar{\mu}}}{H(\bar{\lambda})} p^{\delta}(\bar{\lambda})
$$

is a polynomial in $n$ of degree at most $|\delta|+\ell(\delta)$.

Proof. Let $X(\bar{\lambda})=\left\{x_{0}, x_{1}, \ldots, x_{m}\right\}$ and $Y(\bar{\lambda})=\left\{y_{1}, y_{2}, \ldots, y_{m}\right\}$. First, we show that the difference $p^{k}\left(\bar{\lambda}^{i+}\right)-p^{k}(\bar{\lambda})$ can be written as the following form

$$
\sum_{j=0}^{k} \eta_{j}(\bar{\lambda})\left(\begin{array}{c}
x_{i} \\
2
\end{array}\right)^{j}
$$

for $0 \leq i \leq m$ and a nonnegative integer $k$, where each coefficient $\eta_{j}(\bar{\lambda})$ is a linear combination of some $\Phi^{\tau}(\bar{\lambda})$ for some usual partition $\tau$ of size $|\tau| \leq k$. Indeed, by Lemma 2.3 and Theorem 2.1,

$$
\begin{aligned}
p^{k}\left(\bar{\lambda}^{0+}\right)-p^{k}(\bar{\lambda}) & =2 \sum_{j=1}^{m}\left(x_{j}^{2 k}+\left(x_{j}-1\right)^{2 k}\right)-2 \sum_{j=1}^{m}\left(y_{j}^{2 k}+\left(y_{j}-1\right)^{2 k}\right)+2^{2 k}+1 \\
& =\eta_{0}(\bar{\lambda})=\sum_{j=0}^{k} \eta_{j}(\bar{\lambda})\left(\begin{array}{c}
x_{0} \\
2
\end{array}\right)^{j} \quad\left(\text { if } i=0 \text { and } \bar{\lambda}_{\ell(\bar{\lambda})} \geq 2\right)
\end{aligned}
$$

and

$$
\begin{aligned}
& p^{k}\left(\bar{\lambda}^{i+}\right)-p^{k}(\bar{\lambda}) \\
= & 2 \sum_{j=1}^{m}\left(\left(x_{i}-x_{j}\right)^{2 k}+\left(x_{i}+x_{j}-1\right)^{2 k}\right)-2 \sum_{j=1}^{m}\left(\left(x_{i}-y_{j}\right)^{2 k}+\left(x_{i}+y_{j}-1\right)^{2 k}\right) \\
+ & 2 x_{i}^{2 k}+2\left(2 x_{i}-2\right)^{2 k}+2-2\left(2 x_{i}-1\right)^{2 k}+\left(2^{2 k}-1\right)\left(x_{i}^{2 k}-\left(x_{i}-1\right)^{2 k}\right) \\
= & \sum_{j=0}^{k} \eta_{j}(\bar{\lambda})\left(\begin{array}{c}
x_{i} \\
2
\end{array}\right)^{j} \quad(\text { if } 1 \leq i \leq m) .
\end{aligned}
$$

Next, let $A=\Phi^{\nu}(\bar{\lambda})$ and $B=p^{\delta}(\bar{\lambda})$. We have

$$
\begin{aligned}
& \Delta_{i} A:=\Phi^{\nu}\left(\bar{\lambda}^{i+}\right)-\Phi^{\nu}(\bar{\lambda})=\sum_{(*)} \prod_{s \in U} \Phi^{\nu_{s}}(\bar{\lambda}) \prod_{s^{\prime} \in V}\left(\Phi^{\nu_{s^{\prime}}}\left(\bar{\lambda}^{i+}\right)-\Phi^{\nu_{s^{\prime}}}(\bar{\lambda})\right), \\
& \Delta_{i} B:=p^{\delta}\left(\bar{\lambda}^{i+}\right)-p^{\delta}(\bar{\lambda})=\sum_{(* *)} \prod_{s \in U} p^{\delta_{s}}(\bar{\lambda}) \prod_{s^{\prime} \in V}\left(p^{\delta_{s^{\prime}}}\left(\bar{\lambda}^{i+}\right)-p^{\delta_{s^{\prime}}}(\bar{\lambda})\right),
\end{aligned}
$$

where the sum $(*)$ (resp. $(* *))$ ranges over all pairs $(U, V)$ of positive integer sets such that $U \cup V=\{1,2, \ldots, \ell(\nu)\}$ (resp. $U \cup V=\{1,2, \ldots, \ell(\delta)\}$ ), $U \cap V=\emptyset$ and $V \neq \emptyset$.

Finally, it follows from (2.9) and Theorem 2.1 that

$$
\begin{aligned}
& H(\bar{\lambda}) \bar{D}\left(\frac{p^{\delta}(\bar{\lambda}) \Phi^{\nu}(\bar{\lambda})}{H(\bar{\lambda})}\right) \\
= & \frac{H(\bar{\lambda})}{H\left(\bar{\lambda}^{0+}\right)}\left(p^{\delta}\left(\bar{\lambda}^{0+}\right) \Phi^{\nu}\left(\bar{\lambda}^{0+}\right)-p^{\delta}(\bar{\lambda}) \Phi^{\nu}(\bar{\lambda})\right)
\end{aligned}
$$




$$
\begin{aligned}
& +2 \sum_{i=1}^{m} \frac{H(\bar{\lambda})}{H\left(\bar{\lambda}^{i+}\right)}\left(p^{\delta}\left(\bar{\lambda}^{i+}\right) \Phi^{\nu}\left(\bar{\lambda}^{i+}\right)-p^{\delta}(\bar{\lambda}) \Phi^{\nu}(\bar{\lambda})\right) \\
& =\sum_{0 \leq i \leq m} \frac{\left.\prod_{\substack{1 \leq j \leq m \\
0 \leq j \leq m \\
j \neq i}}\left(\left(\begin{array}{c}
x_{i} \\
2
\end{array}\right)-\left(\begin{array}{c}
y_{j} \\
2
\end{array}\right)\right)-\left(\begin{array}{c}
x_{j} \\
2
\end{array}\right)\right)}{\prod_{0}}\left(p^{\delta}\left(\bar{\lambda}^{i+}\right) \Phi^{\nu}\left(\bar{\lambda}^{i+}\right)-p^{\delta}(\bar{\lambda}) \Phi^{\nu}(\bar{\lambda})\right) \\
& \prod\left(\left(\begin{array}{c}
x_{i} \\
2
\end{array}\right)-\left(\begin{array}{c}
y_{j} \\
2
\end{array}\right)\right)
\end{aligned}
$$

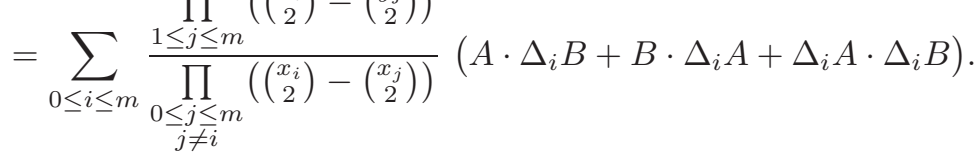

(By Theorem 2.1)

By Theorems 2.4 and 2.2, each of the above three terms could be written as a linear combination of some $p^{\underline{\delta}}(\bar{\lambda}) \Phi \underline{\underline{\nu}}(\bar{\lambda})$ satisfying $|\underline{\delta}|+\ell(\underline{\delta})+|\underline{\nu}| \leq|\delta|+\ell(\delta)+|\nu|-1$. Then the claim follows by induction on $|\delta|+\ell(\delta)+|\nu|$.

When $\bar{\mu}=\emptyset$, the summation (2.13) in Theorem 2.6 becomes

$$
\sum_{|\bar{\lambda}|=n} \frac{2^{n-\ell(\bar{\lambda})} n !}{H(\bar{\lambda})^{2}} p^{\delta}(\bar{\lambda})
$$

or

$$
2^{n} n ! \sum_{|\bar{\lambda} \bar{\lambda}|=2 n} \frac{1}{H(\bar{\lambda} \bar{\lambda})} \Psi^{\delta}\left(\left\{h^{2}: h \in \mathcal{H}(\bar{\lambda} \lambda)\right\}, \emptyset\right)
$$

by (2.1). The above summation is a polynomial in $n$. Consequently, Theorem 1.4 is true when $t=1$ and $Q_{2}=1$. Other specializations are listed as follows.

Theorem 2.7. Let $\bar{\mu}$ be a given strict partition. Then,

$$
\sum_{|\bar{\lambda} / \bar{\mu}|=n} \frac{2^{|\bar{\lambda}|-\ell(\bar{\lambda})-|\bar{\mu}|+\ell(\bar{\mu})} f_{\bar{\lambda} / \bar{\mu}} H(\bar{\mu})}{H(\bar{\lambda})}\left(p^{1}(\bar{\lambda})-p^{1}(\bar{\mu})\right)=12\left(\begin{array}{l}
n \\
2
\end{array}\right)+(12|\bar{\mu}|+5) n .
$$

Let $\bar{\mu}=\emptyset$. We obtain

$$
2^{n} n ! \sum_{|\bar{\lambda} \bar{\lambda}|=2 n} \frac{1}{H(\bar{\lambda} \bar{\lambda})} \sum_{h \in \mathcal{H}(\bar{\lambda} \bar{\lambda})} h^{2}=12\left(\begin{array}{l}
n \\
2
\end{array}\right)+5 n .
$$

Proof. We have

$$
\begin{aligned}
& p^{1}\left(\bar{\lambda}^{0+}\right)-p^{1}(\bar{\lambda})=2 \sum_{j=1}^{m}\left(x_{j}^{2}+\left(x_{j}-1\right)^{2}\right)-2 \sum_{j=1}^{m}\left(y_{j}^{2}+\left(y_{j}-1\right)^{2}\right)+2^{2}+1 \\
= & \eta_{0}(\bar{\lambda})=8|\bar{\lambda}|+5 \quad\left(\text { if } i=0 \text { and } \bar{\lambda}_{\ell(\bar{\lambda})} \geq 2\right)
\end{aligned}
$$

and

$$
\begin{aligned}
& p^{1}\left(\bar{\lambda}^{i+}\right)-p^{1}(\bar{\lambda}) \\
= & 2 \sum_{j=1}^{m}\left(\left(x_{i}-x_{j}\right)^{2}+\left(x_{i}+x_{j}-1\right)^{2}\right)-2 \sum_{j=1}^{m}\left(\left(x_{i}-y_{j}\right)^{2}+\left(x_{i}+y_{j}-1\right)^{2}\right) \\
+ & 2 x_{i}^{2}+2\left(2 x_{i}-2\right)^{2}+2-2\left(2 x_{i}-1\right)^{2}+\left(2^{2}-1\right)\left(x_{i}^{2}-\left(x_{i}-1\right)^{2}\right) \\
= & 4\left(\begin{array}{c}
x_{i} \\
2
\end{array}\right)+8|\bar{\lambda}|+5 \quad \quad(\text { if } 1 \leq i \leq m) .
\end{aligned}
$$


So that

$$
\begin{aligned}
H(\bar{\lambda}) \bar{D}\left(\frac{p^{1}(\bar{\lambda})}{H(\bar{\lambda})}\right) & =\sum_{0 \leq i \leq m} \frac{\prod_{\substack{1 \leq j \leq m \\
0 \leq j \leq m \\
j \neq i}}\left(\left(\begin{array}{c}
x_{i} \\
2
\end{array}\right)-\left(\begin{array}{c}
y_{j} \\
2
\end{array}\right)\right)}{\left.\left.\prod_{2}\right)-\left(\begin{array}{c}
x_{j} \\
2
\end{array}\right)\right)}\left(4\left(\begin{array}{c}
x_{i} \\
2
\end{array}\right)+8|\bar{\lambda}|+5\right) \\
& =4 \Phi^{1}(\bar{\lambda})+8|\bar{\lambda}|+5 \\
& =12|\bar{\lambda}|+5 .
\end{aligned}
$$

Therefore we have

$$
\begin{aligned}
& H(\bar{\lambda}) \bar{D}^{2}\left(\frac{p^{1}(\bar{\lambda})}{H(\bar{\lambda})}\right)=12, \\
& H(\bar{\lambda}) \bar{D}^{3}\left(\frac{p^{1}(\bar{\lambda})}{H(\bar{\lambda})}\right)=0 .
\end{aligned}
$$

Identity (2.16) follows from Theorem 2.5. By (2.1), we derive (2.17).

Recall the following results obtained in [12] involving the contents of strict partitions.

Theorem 2.8. Suppose that $Q$ is a given symmetric function, and $\bar{\mu}$ is a given strict partition. Then

$$
\sum_{|\bar{\lambda} / \bar{\mu}|=n} \frac{2^{|\bar{\lambda}|-|\bar{\mu}|-\ell(\bar{\lambda})+\ell(\bar{\mu})} f_{\bar{\lambda} / \bar{\mu}}}{H(\bar{\lambda})} Q\left(\left(\begin{array}{l}
c \\
2
\end{array}\right): c \in \mathcal{C}(\bar{\lambda})\right)
$$

is a polynomial in $n$.

Theorem 2.9. Suppose that $k$ is a given nonnegative integer. Then

$$
\sum_{|\bar{\lambda}|=n} \frac{2^{|\bar{\lambda}|-\ell(\bar{\lambda})} f_{\bar{\lambda}}}{H(\bar{\lambda})} \sum_{c \in \mathcal{C}(\bar{\lambda})}\left(\begin{array}{c}
c+k-1 \\
2 k
\end{array}\right)=\frac{2^{k}}{(k+1) !}\left(\begin{array}{c}
n \\
k+1
\end{array}\right) .
$$

Theorem 2.10. Let $\bar{\mu}$ be a strict partition. Then,

$$
\sum_{|\bar{\lambda} / \bar{\mu}|=n} \frac{2^{|\bar{\lambda}|-\ell(\bar{\lambda})-|\bar{\mu}|+\ell(\bar{\mu})} f_{\bar{\lambda} / \bar{\mu}} H(\bar{\mu})}{H(\bar{\lambda})}\left(\sum_{c \in \mathcal{C}(\bar{\lambda})}\left(\begin{array}{l}
c \\
2
\end{array}\right)-\sum_{c \in \mathcal{C}(\bar{\mu})}\left(\begin{array}{l}
c \\
2
\end{array}\right)\right)=\left(\begin{array}{l}
n \\
2
\end{array}\right)+n|\bar{\mu}| .
$$

The above results can be interpreted in terms of doubled distinct partitions. In particular, we obtain Theorem 1.4 when $t=1$ and $Q_{1}=1$.

Theorem 2.11. For each usual partition $\delta$, the summation

$$
2^{n} n ! \sum_{|\bar{\lambda} \bar{\lambda}|=2 n} \frac{1}{H(\bar{\lambda} \bar{\lambda})} \Psi^{\delta}(\mathcal{C}(\bar{\lambda} \bar{\lambda}), \emptyset)
$$

is a polynomial in $n$.

Proof. Since $c+(1-c)=1$ and $c(1-c)=-2\left(\begin{array}{c}c \\ 2\end{array}\right)$, there exists some $a_{i}$ such that $c^{k}+(1-c)^{k}=\sum_{i=1}^{s} a_{i}\left(\begin{array}{c}c \\ 2\end{array}\right)^{i}$. By $(2.2)$, we obtain

$$
\sum_{c \in \mathcal{C}(\bar{\lambda} \bar{\lambda})} c^{k}=\sum_{c \in \mathcal{C}(\bar{\lambda})}\left(c^{k}+(1-c)^{k}\right)=\sum_{i=1}^{s} a_{i} \sum_{c \in \mathcal{C}(\bar{\lambda})}\left(\begin{array}{l}
c \\
2
\end{array}\right)^{i} .
$$

The claim follows from Theorem 2.8. 


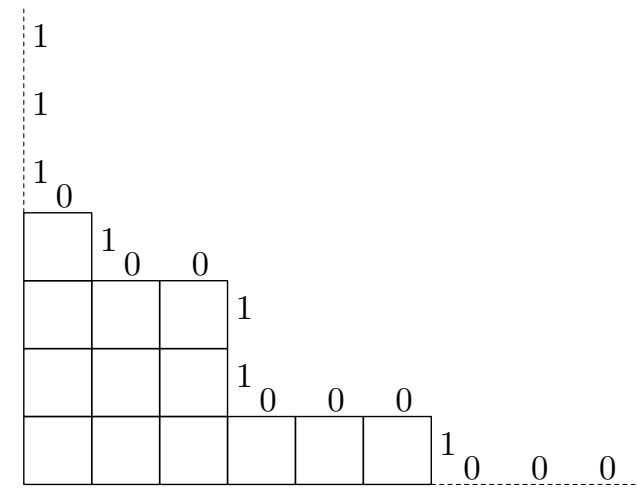

Figure 5. From usual partitions to bi-infinite 01-sequences.

The following results are corollaries of Theorems 2.9 and 2.10.

Theorem 2.12. Suppose that $k$ is a given nonnegative integer. Then,

$$
\begin{aligned}
& 2^{n} n ! \sum_{|\bar{\lambda} \bar{\lambda}|=2 n} \frac{1}{H(\bar{\lambda} \bar{\lambda})} \sum_{c \in \mathcal{C}(\bar{\lambda} \bar{\lambda})}\left(\begin{array}{c}
c+k-1 \\
2 k
\end{array}\right)=\frac{2^{k+1}}{(k+1) !}\left(\begin{array}{c}
n \\
k+1
\end{array}\right), \\
& 2^{n} n ! \sum_{|\bar{\lambda} \bar{\lambda}|=2 n} \frac{1}{H(\bar{\lambda} \bar{\lambda})} \sum_{c \in \mathcal{C}(\bar{\lambda} \bar{\lambda})} c^{2}=4\left(\begin{array}{c}
n \\
2
\end{array}\right)+\left(\begin{array}{c}
n \\
1
\end{array}\right) .
\end{aligned}
$$

\section{The LitTlewood DECOMPOSITION AND CORNERs OF USUAL PARTitions}

In this section we recall some basic definitions and properties for usual partitions (see [10], [17, p.12], [30, p.468], [14, p.75], [6]). Let $\mathcal{W}$ be the set of bi-infinite binary sequences beginning with infinitely many 0 's and ending with infinitely many 1 's. Each element $w$ of $\mathcal{W}$ can be represented by $\left(a_{i}^{\prime}\right)_{i}=\cdots a_{-3}^{\prime} a_{-2}^{\prime} a_{-1}^{\prime} a_{0}^{\prime} a_{1}^{\prime} a_{2}^{\prime} a_{3}^{\prime} \cdots$. However, the representation is not unique, since for any fixed integer $k$ the sequence $\left(a_{i+k}^{\prime}\right)_{i}$ also represents $w$. The canonical representation of $w$ is the unique sequence $\left(a_{i}\right)_{i}=\cdots a_{-3} a_{-2} a_{-1} a_{0} a_{1} a_{2} a_{3} \cdots$ such that

$$
\#\left\{i \leq-1, a_{i}=1\right\}=\#\left\{i \geq 0, a_{i}=0\right\} .
$$

It will be further denoted by $\cdots a_{-3} a_{-2} a_{-1} . a_{0} a_{1} a_{2} a_{3} \cdots$ with a dot symbol inserted between the letters $a_{-1}$ and $a_{0}$. There is a one to one correspondence described as follow between $\mathcal{W}$ and the set of partitions $\mathcal{P}$ (see, e.g. [30, p.468], [1] for more details). Let $\lambda$ be a partition. We encode each horizontal edge of $\lambda$ by 1 and each vertical edge by 0 . Reading these $(0,1)$-encodings from top to bottom and from left to right yields a binary word $u$. By adding infinitely many 0's to the left and infinitely many 1 's to the right of $u$ we get an element $w=\cdots 000 u 111 \cdots \epsilon$ $\mathcal{W}$. Clearly, the map $\lambda \mapsto w$ is a one-to-one correspondence between $\mathcal{P}$ and $\mathcal{W}$. For example, take $\lambda=(6,3,3,1)$. Then $u=0100110001$, so that $w=\left(a_{i}\right)_{i}=$ $\cdots 1110100.110001000 \cdots$ (see Figure 5).

Let $t$ be a positive integer. Recall that a partition $\lambda$ is a $t$-core if it has no hook equal to $t$. The set of all $t$-core partitions (resp. $t$-core doubled distinct partitions) are denoted by $\mathcal{P}_{t \text {-core }}$ (resp. $\left.\mathcal{D} \mathcal{D}_{t \text {-core }}\right)$. The Littlewood decomposition 


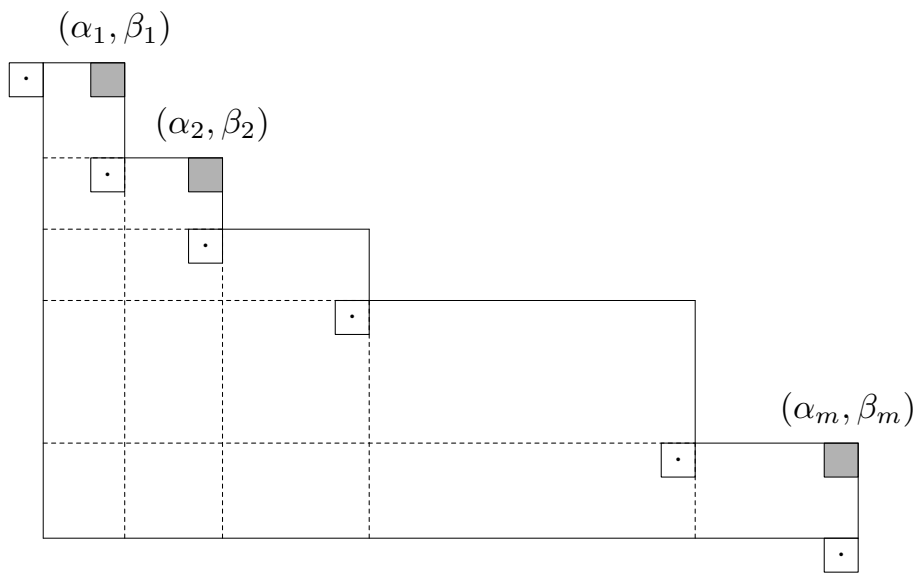

FiguRE 6. A partition and its corners. The outer corners are labelled with $\left(\alpha_{i}, \beta_{i}\right)(i=1,2, \ldots, m)$. The inner corners are indicated by the dot symbol ".".

maps bijectively a usual partition $\lambda$ to $\left(\lambda_{t \text {-core }} ; \lambda^{0}, \lambda^{1}, \ldots, \lambda^{t-1}\right) \in \mathcal{P}_{t \text {-core }} \times \mathcal{P}^{t}$ such that

(P1) $\lambda_{t \text {-core }}$ is a $t$-core and $\lambda^{0}, \lambda^{1}, \ldots, \lambda^{t-1}$ are usual partitions;

(P2) $|\lambda|=\left|\lambda_{t \text {-core }}\right|+t\left(\left|\lambda^{0}\right|+\left|\lambda^{1}\right|+\cdots+\left|\lambda^{t-1}\right|\right)$;

(P3) $\left\{h / t \mid h \in \mathcal{H}_{t}(\lambda)\right\}=\mathcal{H}\left(\lambda^{0}\right) \cup \mathcal{H}\left(\lambda^{1}\right) \cup \cdots \cup \mathcal{H}\left(\lambda^{t-1}\right)$.

The vector $\left(\lambda^{0}, \lambda^{1}, \ldots, \lambda^{t-1}\right)$ is called the $t$-quotient of the partition $\lambda$.

It is well know that (see [6]) under the Littlewood decomposition, a doubled distinct partition $\lambda$ has image $\left(\lambda_{t \text {-core }} ; \lambda^{0}, \lambda^{1}, \ldots, \lambda^{t-1}\right) \in \mathcal{D} \mathcal{D}_{t \text {-core }} \times \mathcal{D} \mathcal{D} \times \mathcal{P}^{t-1}$ where $\lambda^{i}$ is the conjugate partition of $\lambda^{t-i}$ for $1 \leq i \leq t-1$.

Recall that we define in Section 2 the notion of inner corners $x_{i}(0 \leq i \leq m)$ and outer corners $y_{j}(1 \leq j \leq m)$ for strict partitions. We use here the same notation for usual partitions (see Figure 6) as in this section. For usual partitions, it is easy to verify that $x_{i}$ and $y_{j}$ satisfy the following relation (see [11, 2]):

$$
x_{0}<y_{1}<x_{1}<y_{2}<x_{2}<\cdots<y_{m}<x_{m} .
$$

Define (see [11])

$$
\Psi^{\nu}(\lambda):=\Psi^{\nu}\left(\left\{x_{i}\right\},\left\{y_{j}\right\}\right)
$$

for each usual partition $\nu$.

Lemma 3.1. Suppose that $\lambda$ is a partition whose set of contents of inner corners and set of contents of outer corners are $X(\lambda)=\left\{x_{0}, x_{1}, \ldots, x_{m}\right\}$ and $Y(\lambda)=$ $\left\{y_{1}, y_{2}, \ldots, y_{m}\right\}$ respectively. Let $\lambda^{i+}=\lambda \cup\left\{\square_{i}\right\}$ where $c_{\square_{i}}=x_{i}$. Then we have

$$
X\left(\lambda^{i+}\right) \cup\left\{x_{i}, x_{i}\right\} \cup Y(\lambda)=X(\lambda) \cup\left\{x_{i}+1, x_{i}-1\right\} \cup Y\left(\lambda^{i+}\right) .
$$

Proof. Four cases are to be considered. (i) If $\beta_{i}+1<\beta_{i+1}$ and $\alpha_{i+1}+1<\alpha_{i}$. Then, the contents of inner corners and outer corners of $\lambda^{i+}$ are $X \cup\left\{x_{i}-1, x_{i}+1\right\} \backslash\left\{x_{i}\right\}$ and $Y \cup\left\{x_{i}\right\}$ respectively. (ii) If $\beta_{i}+1=\beta_{i+1}$ and $\alpha_{i+1}+1<\alpha_{i}$, so that $y_{i+1}=x_{i}+1$. Hence the contents of inner corners and outer corners of $\lambda^{i+}$ are $X \cup\left\{x_{i}-1\right\} \backslash\left\{x_{i}\right\}$ 
and $Y \cup\left\{x_{i}\right\} \backslash\left\{x_{i}+1\right\}$ respectively. (iii) If $\beta_{i}+1<\beta_{i+1}$ and $\alpha_{i+1}+1=\alpha_{i}$, so that $y_{i}=x_{i}-1$. Then the contents of inner corners and outer corners of $\lambda^{i+}$ are $X \cup\left\{x_{i}+1\right\} \backslash\left\{x_{i}\right\}$ and $Y \cup\left\{x_{i}\right\} \backslash\left\{x_{i}-1\right\}$ respectively. (iv) If $\beta_{i}+1=\beta_{i+1}$ and $\alpha_{i+1}+1=\alpha_{i}$. Then $y_{i}+1=x_{i}=y_{i+1}-1$. The contents of inner corners and outer corners of $\lambda^{i+}$ are $X \backslash\left\{x_{i}\right\}$ and $Y \cup\left\{x_{i}\right\} \backslash\left\{x_{i}-1, x_{i}+1\right\}$ respectively. The claim is proved.

The corners of the strict partition $\bar{\lambda}$ and the doubled distinct partition $\bar{\lambda} \bar{\lambda}$ are closely related.

Lemma 3.2. Suppose that $\bar{\lambda}$ is a strict partition whose set of contents of inner corners and set of contents of outer corners are $X(\bar{\lambda})=\left\{x_{0}, x_{1}, \ldots, x_{m}\right\}$ and $Y(\bar{\lambda})=\left\{y_{1}, y_{2}, \ldots, y_{m}\right\}$ respectively. Then,

$$
X(\bar{\lambda} \bar{\lambda}) \cup\left\{y_{1}, 1-y_{1}, \ldots, y_{m}, 1-y_{m}\right\}=Y(\bar{\lambda} \bar{\lambda}) \cup\left\{0, x_{1}, 1-x_{1}, \ldots, x_{m}, 1-x_{m}\right\} .
$$

Proof. Two cases are to be considered. (i) If $y_{1}=1$, the contents of inner corners and outer corners of $\bar{\lambda} \bar{\lambda}$ are $X(\bar{\lambda} \bar{\lambda})=\left\{x_{1}, 1-x_{1}, \ldots, x_{m}, 1-x_{m}\right\}$ and $Y(\bar{\lambda} \bar{\lambda})=$ $\left\{1, y_{2}, 1-y_{2} \ldots, y_{m}, 1-y_{m}\right\}$ respectively. (ii) If $y_{1} \geq 2$, the contents of inner corners and outer corners of $\bar{\lambda} \bar{\lambda}$ are $X(\bar{\lambda} \bar{\lambda})=\left\{0, x_{1}, 1-x_{1}, \ldots, x_{m}, 1-x_{m}\right\}$ and $Y(\bar{\lambda} \bar{\lambda})=$ $\left\{y_{1}, 1-y_{1}, \ldots, y_{m}, 1-y_{m}\right\}$ respectively. This achieves the proof of Lemma 3.2.

\section{The $t$-DIFFERENCE OPERATORS FOR DOUbled DistinCt PARTITIONS}

Let $t=2 t^{\prime}+1$ be an odd positive integer. For each strict partition $\bar{\lambda}$, the doubled distinct partition associated with $\bar{\lambda}$ is denoted by $\lambda=\bar{\lambda} \bar{\lambda}$. The Littlewood decomposition maps $\bar{\lambda} \bar{\lambda}$ to (see [6])

$$
\left(\lambda_{t \text {-core }} ; \lambda^{0}, \lambda^{1}, \ldots, \lambda^{2 t^{\prime}}\right) \in \mathcal{D} \mathcal{D}_{t \text {-core }} \times \mathcal{D} \mathcal{D} \times \mathcal{P}^{2 t^{\prime}}
$$

where $\lambda^{i}$ is the conjugate partition of $\lambda^{t-i}$ for $1 \leq i \leq t^{\prime}$. For convenience we say that the Littlewood decomposition maps the strict partition $\bar{\lambda}$ to

$$
\bar{\lambda} \mapsto\left(\bar{\lambda}_{t \text {-core }} ; \bar{\lambda}^{0}, \lambda^{1}, \ldots, \lambda^{t^{\prime}}\right),
$$

where $\bar{\lambda}_{t \text {-core }}$ and $\bar{\lambda}^{0}$ are determined by $\lambda_{t \text {-core }}=\bar{\lambda}_{t \text {-core }} \bar{\lambda}_{t \text {-core }}$ and $\lambda^{0}=\bar{\lambda}^{0} \bar{\lambda}^{0}$.

Example 4.1. Let $\bar{\lambda}=(6,5,4,1)$ and $t=3$. Then $\lambda=\bar{\lambda} \bar{\lambda}=(7,7,7,5,3,3)$. The bi-infinite binary sequence of $\lambda$ is $\cdots 111000110.0100111000 \cdots$. By Littlewood decomposition (see [30, p.468], [1]), the bi-infinite binary sequence of $\lambda_{t \text {-core }}$, $\lambda^{0}, \lambda^{1}, \lambda^{2}$ are $\cdots 1110.01000 \cdots, \cdots 11101.001000 \cdots, \cdots 11101.110000 \cdots$ and $\cdots 11100.01000 \cdots$, respectively, which means that $\lambda_{t \text {-core }}=(2), \lambda^{0}=(3,1), \lambda^{1}=$ $(1,1,1), \lambda^{2}=(3), \bar{\lambda}_{t \text {-core }}=(1)$ and $\bar{\lambda}^{0}=(2)$. Therefore, $\bar{\lambda}=(6,5,4,1)$ is mapped to $((1) ;(2),(1,1,1))$ in $(4.1)$.

Since the map (4.1) is bijective, we always write

$$
\lambda=\left(\bar{\lambda}_{t \text {-core }} ; \bar{\lambda}^{0}, \lambda^{1}, \ldots, \lambda^{t^{\prime}}\right) .
$$

Let $\lambda=\bar{\lambda} \bar{\lambda}=\left(\bar{\lambda}_{t \text {-core }} ; \bar{\lambda}^{0}, \lambda^{1}, \ldots, \lambda^{t^{\prime}}\right)$ and $\mu=\bar{\mu} \bar{\mu}=\left(\bar{\mu}_{t \text {-core }} ; \bar{\mu}^{0}, \mu^{1}, \ldots, \mu^{t^{\prime}}\right)$ be two doubled distinct partitions. If $\lambda_{t \text {-core }}=\mu_{t \text {-core }}, \bar{\lambda}^{0} \supset \bar{\mu}^{0}$ and $\lambda^{i} \supset \mu^{i}$ for $1 \leq i \leq t^{\prime}$, we write $\lambda \geq_{t} \mu$ and define

$$
F_{\mu / \mu}^{\mathcal{D D}}:=1 \quad \text { and } \quad F_{\lambda / \mu}^{\mathcal{D D}}:=\sum_{\substack{\lambda \geq t \lambda^{-} \geq_{t} \mu \\\left|\lambda / \lambda^{-}\right|=2 t}} F_{\lambda^{-} / \mu}^{\mathcal{D D}} \quad(\text { for } \lambda \neq \mu) .
$$


In fact, $F_{\lambda / \mu}^{\mathcal{D D}}$ is the number of vectors $\left(P_{0}, P_{1}, \ldots, P_{t^{\prime}}\right)$ such that

(1) $P_{0}$ is a skew shifted Young tableau of shape $\bar{\lambda}^{0} / \bar{\mu}^{0}$,

(2) $P_{i}\left(1 \leq i \leq t^{\prime}\right)$ is a skew Young tableau of shape $\lambda^{i} / \mu^{i}$,

(3) the union of entries in $P_{0}, P_{1}, \ldots, P_{t^{\prime}}$ are $1,2, \ldots,\left|\bar{\lambda}^{0} / \bar{\mu}^{0}\right|+\sum_{i=1}^{t^{\prime}}\left|\lambda^{i} / \mu^{i}\right|$. Hence,

$$
F_{\lambda / \mu}^{\mathcal{D D}}=\left(\begin{array}{c}
\left|\bar{\lambda}^{0} / \bar{\mu}^{0}\right|+\sum_{i=1}^{t^{\prime}}\left|\lambda^{i} / \mu^{i}\right| \\
\left|\bar{\lambda}^{0} / \bar{\mu}^{0}\right|,\left|\lambda^{1} / \mu^{1}\right|, \ldots,\left|\lambda^{t^{\prime}} / \mu^{t^{\prime}}\right|
\end{array}\right) f_{\bar{\lambda}^{0} / \bar{\mu}^{0}} \prod_{i=1}^{t^{\prime}} f_{\lambda^{i} / \mu^{i}}
$$

We set

$$
F_{\lambda}^{\mathcal{D D}}:=F_{\lambda / \lambda_{t-\text { core }}}^{\mathcal{D D}}=\left(\begin{array}{c}
\left|\bar{\lambda}^{0}\right|+\sum_{i=1}^{t^{\prime}}\left|\lambda^{i}\right| \\
\left|\bar{\lambda}^{0}\right|,\left|\lambda^{1}\right|, \ldots,\left|\lambda^{t^{\prime}}\right|
\end{array}\right) f_{\bar{\lambda}^{0}} \prod_{i=1}^{t^{\prime}} f_{\lambda^{i}}=\frac{n !}{H\left(\bar{\lambda}^{0}\right) \prod_{i=1}^{t^{\prime}} H\left(\lambda^{i}\right)}
$$

and

$$
G_{\lambda}^{\mathcal{D D}}:=\frac{2^{n-\ell\left(\bar{\lambda}^{0}\right)}}{t^{n} H\left(\bar{\lambda}^{0}\right) \prod_{i=1}^{t^{\prime}} H\left(\lambda^{i}\right)}=\frac{2^{n-\ell\left(\bar{\lambda}^{0}\right)} F_{\lambda}^{\mathcal{D D}}}{t^{n} n !}
$$

where $n=\left|\bar{\lambda}^{0}\right|+\sum_{i=1}^{t^{\prime}}\left|\lambda^{i}\right|$.

When $t=1$, we have $t^{\prime}=0$, thus $F_{\lambda}^{\mathcal{D D}}=f_{\bar{\lambda}^{0}}$ and $G_{\lambda}^{\mathcal{D D}}=2^{n-\ell\left(\bar{\lambda}^{0}\right)} / H\left(\bar{\lambda}^{0}\right)$. Also, when $\lambda$ is a $t$-core doubled distinct partition, we have $F_{\lambda}^{\mathcal{D D}}=G_{\lambda}^{\mathcal{D D}}=1$.

4.1. $t$-difference operators. Let $g: \mathcal{D D} \rightarrow \mathbb{R}$ be a function of doubled distinct partitions and $\lambda$ be a doubled distinct partition. The t-difference operator $D_{t}^{\mathcal{D D}}$ for doubled distinct partitions is defined by

$$
D_{t}^{\mathcal{D D}} g(\lambda)=\sum_{\substack{\lambda^{+} \geq t \lambda \\\left|\lambda^{+} / \lambda\right|=2 t}} g\left(\lambda^{+}\right)-g(\lambda)
$$

The higher-order $t$-difference operators $D_{t}^{k}$ are defined by induction:

$$
\left(D_{t}^{\mathcal{D D}}\right)^{0} g:=g \quad \text { and } \quad\left(D_{t}^{\mathcal{D D}}\right)^{k} g:=D_{t}^{\mathcal{D D}}\left(\left(D_{t}^{\mathcal{D D}}\right)^{k-1} g\right) \quad(k \geq 1) .
$$

Lemma 4.1. Let $\lambda$ be a doubled distinct partition. Then,

$$
D_{t}^{\mathcal{D D}}\left(G_{\lambda}^{\mathcal{D D}}\right)=0
$$

In other words,

$$
G_{\lambda}^{\mathcal{D D}}=\sum_{\substack{\lambda^{+} \geq t \lambda \\\left|\lambda^{+} / \lambda\right|=2 t}} G_{\lambda^{+}}^{\mathcal{D D}}
$$

Proof. Write $\lambda=\left(\bar{\lambda}_{t \text {-core }} ; \bar{\lambda}^{0}, \lambda^{1}, \ldots, \lambda^{t^{\prime}}\right)$. By Theorem 3.3 in [12] we obtain

$$
\sum_{\left|\left(\bar{\lambda}^{0}\right)^{+} / \bar{\lambda}^{0}\right|=1} \frac{G_{\left(\bar{t}_{t-\text { core }} ;\left(\bar{\lambda}^{0}\right)^{+}, \lambda^{1}, \ldots, \lambda^{t^{\prime}}\right)}^{\mathcal{D} \mathcal{D}}}{G_{\lambda}^{\mathcal{D D}}}=\sum_{\left|\left(\bar{\lambda}^{0}\right)^{+} / \bar{\lambda}^{0}\right|=1} \frac{2^{1+\ell\left(\bar{\lambda}^{0}\right)-\ell\left(\left(\bar{\lambda}^{0}\right)^{+}\right)} H\left(\bar{\lambda}^{0}\right)}{t H\left(\left(\bar{\lambda}^{0}\right)^{+}\right)}=\frac{1}{t} .
$$

For $1 \leq i \leq t^{\prime}$, we derive

$$
\sum_{\left|\left(\lambda^{i}\right)^{+} / \lambda^{i}\right|=1} \frac{G_{\left(\bar{\lambda}_{t-\text { core }} ; \bar{\lambda}^{0}, \lambda^{1}, \ldots, \lambda^{i-1},\left(\lambda^{i}\right)^{+}, \lambda^{i+1}, \ldots, \lambda^{t^{\prime}}\right)}^{\mathcal{D P}}}{G_{\lambda}^{\mathcal{D D}}}=\sum_{\left|\left(\lambda^{i}\right)^{+} / \lambda^{i}\right|=1} \frac{2 H\left(\lambda^{i}\right)}{t H\left(\left(\lambda^{i}\right)^{+}\right)}=\frac{2}{t}
$$


by Lemma 2.2 in [11]. Summing the above equalities, we get

$$
\sum_{\substack{\lambda^{+} \geq t \lambda \\\left|\lambda^{+} / \lambda\right|=2 t}} \frac{G_{\lambda^{+}}^{\mathcal{D D}}}{G_{\lambda}^{\mathcal{D} \mathcal{D}}}=\frac{1}{t}+\sum_{i=1}^{t^{\prime}} \frac{2}{t}=1
$$

Lemma 4.2. Suppose that $\mu$ is a given doubled distinct partition and $g: \mathcal{D D} \rightarrow \mathbb{R}$ is a function of doubled distinct partitions. For every $n \in \mathbb{N}$, let

$$
P(\mu, g ; n):=\sum_{\substack{\lambda \in \mathcal{D} \mathcal{D}, \lambda \geq t \mu \\|\lambda / \mu|=2 n t}} F_{\lambda / \mu}^{\mathcal{D} \mathcal{D}} g(\lambda)
$$

Then

$$
P(\mu, g ; n+1)-P(\mu, g ; n)=P\left(\mu, D_{t}^{\mathcal{D D}} g ; n\right) .
$$

Proof. The proof is straightforward:

$$
\begin{aligned}
& P(\mu, g ; n+1)-P(\mu, g ; n)=\sum_{\substack{\nu \geq t \mu \\
|\nu / \mu|=2(n+1) t}} F_{\nu / \mu}^{\mathcal{D D}} g(\nu)-\sum_{\substack{\lambda \geq t \mu \\
|\lambda / \mu|=2 n t}} F_{\lambda / \mu}^{\mathcal{D D}} g(\lambda) \\
& =\sum_{\substack{\nu \geq t \mu \\
|\nu / \mu|=2(n+1) t}} \sum_{\substack{\nu \geq t \nu^{-} \geq t \mu \\
\left|\nu / \nu^{-}\right|=2 t}} F_{\nu^{-} / \mu}^{\mathcal{D D}} g(\nu)-\sum_{\substack{\lambda \geq t \mu \\
|\lambda / \mu|=2 n t}} F_{\lambda / \mu}^{\mathcal{D D}} g(\lambda) \\
& =\sum_{\substack{\lambda \geq t \mu \\
|\lambda / \mu|=2 n t}} F_{\lambda / \mu}^{\mathcal{D D}}\left(\sum_{\substack{\lambda^{+} \geq t \lambda \\
\left|\lambda^{+} / \lambda\right|=2 t}} g\left(\lambda^{+}\right)-g(\lambda)\right) \\
& =P\left(\mu, D_{t}^{\mathcal{D D}} g ; n\right) .
\end{aligned}
$$

Example 4.2. Let $g(\lambda)=G_{\lambda}^{\mathcal{D D}}$. Then $D_{t}^{\mathcal{D D}} g(\lambda)=0$ by Lemma 4.1, which means that $P\left(\mu, D_{t}^{\mathcal{D D}} g ; n\right)=0$. Consequently, $P\left(\mu, G_{\lambda}^{\mathcal{D D}} ; n+1\right)=P\left(\mu, G_{\lambda}^{\mathcal{D D}} ; n\right)=\cdots=$ $P\left(\mu, G_{\lambda}^{\mathcal{D D}} ; 0\right)=G_{\mu}^{\mathcal{D D}}$, or

$$
\sum_{\substack{\lambda \in \mathcal{D} \mathcal{D}, \lambda \geq t \mu \\|\lambda / \mu|=2 n t}} F_{\lambda / \mu}^{\mathcal{D D}} G_{\lambda}^{\mathcal{D D}}=G_{\mu}^{\mathcal{D D}}
$$

When $\mu$ is a $t$-core doubled distinct partition, the above identity becomes

$$
\sum_{\substack{\lambda \geq t \mu \\|\lambda / \mu|=2 n t}} \frac{n !}{H\left(\bar{\lambda}^{0}\right) \prod_{i=1}^{t^{\prime}} H\left(\lambda^{i}\right)} \times \frac{2^{n-\ell\left(\bar{\lambda}^{0}\right)}}{t^{n} H\left(\bar{\lambda}^{0}\right) \prod_{i=1}^{t^{\prime}} H\left(\lambda^{i}\right)}=G_{\mu}^{\mathcal{D D}},
$$

or

$$
\sum_{\substack{\lambda \in \mathcal{D} \mathcal{D},|\lambda / \mu|=2 n t \\ \lambda \geq_{t} \mu}} \frac{(2 t)^{n} n !}{H_{t}(\lambda)}=1
$$

which implies (1.2).

Theorem 4.3. Let $g: \mathcal{D D} \rightarrow \mathbb{R}$ be a function of doubled distinct partitions and $\mu$ be a given doubled distinct partition. Then,

$$
P(\mu, g ; n)=\sum_{\substack{\lambda \in \mathcal{D} \mathcal{D}, \lambda \geq t \mu \\
|\lambda / \mu|=2 n t}} F_{\lambda / \mu}^{\mathcal{D} \mathcal{D}} g(\lambda)=\sum_{k=0}^{n}\left(\begin{array}{l}
n \\
k
\end{array}\right)\left(D_{t}^{\mathcal{D} \mathcal{D}}\right)^{k} g(\mu)
$$


and

$$
\left(D_{t}^{\mathcal{D D}}\right)^{n} g(\mu)=\sum_{k=0}^{n}(-1)^{n+k}\left(\begin{array}{l}
n \\
k
\end{array}\right) P(\mu, g ; k) .
$$

In particular, if there exists some positive integer $r$ such that $\left(D_{t}^{\mathcal{D D}}\right)^{r} g(\lambda)=0$ for every doubled distinct partition $\lambda \geq_{t} \mu$, then $P(\mu, g ; n)$ is a polynomial in $n$ with degree at most $r-1$.

Proof. Identity (4.7) is proved by induction. The case $n=0$ is obvious. Assume that (4.7) is true for some nonnegative integer $n$. By Lemma 4.2 we obtain

$$
\begin{aligned}
P(\mu, g ; n+1) & =P(\mu, g ; n)+P\left(\mu, D_{t}^{\mathcal{D D}} g ; n\right) \\
& =\sum_{k=0}^{n}\left(\begin{array}{c}
n \\
k
\end{array}\right)\left(D_{t}^{\mathcal{D D}}\right)^{k} g(\mu)+\sum_{k=0}^{n}\left(\begin{array}{l}
n \\
k
\end{array}\right)\left(D_{t}^{\mathcal{D D}}\right)^{k+1} g(\mu) \\
& =\sum_{k=0}^{n+1}\left(\begin{array}{c}
n+1 \\
k
\end{array}\right)\left(D_{t}^{\mathcal{D D}}\right)^{k} g(\mu) .
\end{aligned}
$$

Identity (4.8) follows from the famous Möbius inversion formula [27].

4.2. $\mu$-admissible functions of doubled distinct partitions. Let $\mu=\bar{\mu} \bar{\mu}$ be a $t$-core doubled distinct partition. A function $g: \mathcal{D D} \rightarrow \mathbb{R}$ of doubled distinct partitions is called $\mu$-admissible, if for each given $1 \leq i \leq t^{\prime}$ (resp. $i=0$ ), $g\left(\lambda^{+}\right)-$ $g(\lambda)$ is a polynomial in $c_{\square_{i}}$ (resp. $\left(\begin{array}{c}c^{\square} \\ 2\end{array}\right)$ ) for every pair of partitions

$$
\lambda=\left(\bar{\mu} ; \bar{\lambda}^{0}, \lambda^{1}, \ldots, \lambda^{t^{\prime}}\right)
$$

and

$$
\begin{aligned}
\lambda^{+}= & \left(\bar{\mu} ; \bar{\lambda}^{0}, \lambda^{1}, \ldots, \lambda^{i-1}, \lambda^{i} \cup\left\{\square_{i}\right\}, \lambda^{i+1}, \ldots, \lambda^{t^{\prime}}\right) \\
& \left(\text { resp. } \lambda^{+}=\left(\bar{\mu} ; \bar{\lambda}^{0} \cup\left\{\square_{0}\right\}, \lambda^{1}, \ldots, \lambda^{t^{\prime}}\right)\right),
\end{aligned}
$$

whose coefficients are of form

$$
\sum K\left(\mu, i ; \tau^{0}, \tau^{1}, \ldots, \tau^{t^{\prime}}\right) \Phi^{\tau^{0}}\left(\bar{\lambda}^{0}\right) \prod_{j=1}^{t^{\prime}} \Psi^{\tau^{j}}\left(\lambda^{j}\right),
$$

where the summation is taken over the set of $\left(t^{\prime}+1\right)$-tuple of usual partitions $\left(\tau^{0}, \tau^{1}, \ldots, \tau^{t^{\prime}}\right)$ and $K$ is some function from the set $\mathcal{P} \times \mathbb{N} \times \mathcal{P}^{t^{\prime}+1}$ to the set $\mathbb{R}$.

Lemma 4.4. Let $\mu$ be a t-core doubled distinct partition. Then, the two functions of doubled distinct partitions $\sum_{h \in \mathcal{H}(\lambda)} h^{2 r}$ and $\sum_{c \in \mathcal{C}(\lambda)} c^{r}$ are $\mu$-admissible for any nonnegative integer $r$.

To prove Lemma 4.4, we recall some results on the multisets of hook lengths and contents, obtained in [4]. Suppose that a given $t$-core partition $\mu$ has 01-sequence $w(\mu)=\left(a_{\mu, j}\right)_{j \in \mathbb{Z}}$. For $0 \leq i \leq t-1$ we define [4]

$$
b_{i}:=b_{i}(\mu)=\min \left\{j \in \mathbb{Z}: j \equiv i(\bmod t), a_{\mu, j}=1\right\} .
$$

Lemma 4.5 (Lemma 5.3 of [4]). Let $\lambda$ be a partition and $\left(\lambda_{t \text {-core }} ; \lambda^{0}, \lambda^{1}, \ldots, \lambda^{t-1}\right)$ be the image of the Littlewood decomposition of $\lambda$. Then,

$$
\mathcal{C}(\lambda) \backslash \mathcal{C}\left(\lambda_{t \text {-core }}\right)=\bigcup_{i=0}^{t-1}\left\{t c+b_{i}\left(\lambda_{t-\text { core }}\right)-j: 0 \leq j \leq t-1, c \in \mathcal{C}\left(\lambda^{i}\right)\right\} .
$$


Lemma 4.6 (Lemma 5.4 of [4]). Let $0 \leq i \leq t-1, \lambda$ and $\lambda^{+}$be two usual partitions whose images of the Littlewood decomposition are $\left(\lambda_{t \text {-core }} ; \lambda^{0}, \lambda^{1}, \ldots, \lambda^{t-1}\right)$ and

$$
\left(\lambda_{t \text {-core }} ; \lambda^{0}, \lambda^{1}, \ldots, \lambda^{i-1}, \lambda^{i} \cup\left\{\square_{i}\right\}, \lambda^{i+1}, \ldots, \lambda^{t-1}\right)
$$

respectively. Write $b_{j}=b_{j}\left(\lambda_{t \text {-core }}\right)(0 \leq j \leq t-1)$. Assume that $r$ is a given integer and $k$ is an integer satisfying $1 \leq k \leq t-1$. Let $x_{j, s}\left(0 \leq s \leq m_{j}\right)$ be the contents of inner corners of $\lambda^{j}$ and $y_{j, s}\left(1 \leq s \leq m_{j}\right)$ be the contents of outer corners of $\lambda^{j}$ for $0 \leq j \leq t-1$. We have

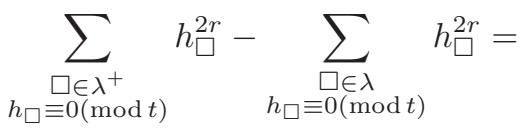

$$
\begin{aligned}
& t^{2 r}+\sum_{0 \leq s \leq m_{i}}\left(t\left(c_{\square_{i}}-x_{i, s}\right)\right)^{2 r}-\sum_{1 \leq s \leq m_{i}}\left(t\left(c_{\square_{i}}-y_{i, s}\right)\right)^{2 r}
\end{aligned}
$$

and

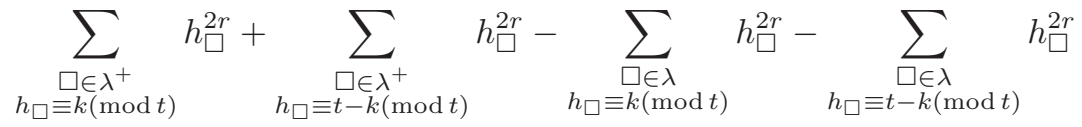

$$
\begin{aligned}
& =\sum_{0 \leq s \leq m_{i^{\prime}}}\left(t c_{\square_{i}}+b_{i}-t x_{i^{\prime}, s}-b_{i^{\prime}}\right)^{2 r}-\sum_{1 \leq s \leq m_{i^{\prime}}}\left(t c_{\square_{i}}+b_{i}-t y_{i^{\prime}, s}-b_{i^{\prime}}\right)^{2 r} \\
& +\sum_{0 \leq s \leq m_{i^{\prime \prime}}}\left(t c_{\square_{i}}+b_{i}-t x_{i^{\prime \prime}, s}-b_{i^{\prime \prime}}\right)^{2 r}-\sum_{1 \leq s \leq m_{i^{\prime \prime}}}\left(t c_{\square_{i}}+b_{i}-t y_{i^{\prime \prime}, s}-b_{i^{\prime \prime}}\right)^{2 r}
\end{aligned}
$$

where $0 \leq i^{\prime}, i^{\prime \prime} \leq t-1$ satisfy $i^{\prime} \equiv i+k(\bmod t)$ and $i^{\prime \prime} \equiv i-k(\bmod t)$. Furthermore,

$$
\begin{aligned}
\sum_{\square \in \lambda^{+}} h_{\square}^{2 r}-\sum_{\square \in \lambda} h_{\square}^{2 r} & =t^{2 r}+\sum_{j=0}^{t-1}\left(\sum_{0 \leq s \leq m_{j}}\left(t c_{\square_{i}}+b_{i}-t x_{j, s}-b_{j}\right)^{2 r}\right. \\
& \left.-\sum_{1 \leq s \leq m_{j}}\left(t c_{\square_{i}}+b_{i}-t y_{j, s}-b_{j}\right)^{2 r}\right) .
\end{aligned}
$$

For the doubled distinct partition $\lambda$ whose image under Littlewood decomposition is $\left(\lambda_{t \text {-core }} ; \lambda^{0}, \lambda^{1}, \ldots, \lambda^{t-1}\right)$ where $\lambda^{0}=\bar{\lambda}^{0} \bar{\lambda}^{0}$, let $x_{0, s}\left(0 \leq s \leq m_{0}\right)$ be the contents of inner corners of $\bar{\lambda}^{0}$ and $y_{0, s}\left(1 \leq s \leq m_{0}\right)$ be the contents of outer corners of $\bar{\lambda}^{0}$. Let $x_{i, s}\left(0 \leq s \leq m_{i}\right)$ be the contents of inner corners of $\lambda^{i}$ and $y_{i, s}\left(1 \leq s \leq m_{i}\right)$ be the contents of outer corners of $\lambda^{i}$ for $1 \leq i \leq t-1$. Then $x_{i, s}=-x_{t-i, m_{i}-s}$ and $y_{i, s}=-y_{t-i, m_{i}+1-s}$ since $\lambda^{i}$ and $\lambda^{t-i}$ are conjugate to each other for $1 \leq i \leq t-1$.

Proof of Lemma 4.4. Let $\lambda=\left(\bar{\lambda}_{t \text {-core }} ; \bar{\lambda}^{0}, \lambda^{1}, \ldots, \lambda^{t^{\prime}}\right)$ be a doubled distinct partition and $b_{j}=b_{j}\left(\bar{\lambda}_{t \text {-core }} \bar{\lambda}_{t \text {-core }}\right)$ for $0 \leq j \leq t-1$. The following statements are consequences of Lemma 4.5.

$(C 1)$ Let $1 \leq i \leq t^{\prime}$ and $\lambda^{+}=\left(\bar{\lambda}_{t \text {-core }} ; \bar{\lambda}^{0}, \lambda^{1}, \ldots, \lambda^{i-1},\left(\lambda^{i}\right)^{+}, \lambda^{i+1}, \ldots, \lambda^{t^{\prime}}\right)$ be a doubled distinct partition such that $\left(\lambda^{i}\right)^{+}=\lambda^{i} \cup\left\{\square_{i}\right\}$. We have

$\mathcal{C}\left(\lambda^{+}\right) \backslash \mathcal{C}(\lambda)=\left\{t c_{\square_{i}}+b_{i}-j: 0 \leq j \leq t-1\right\} \cup\left\{-t c_{\square_{i}}+b_{t-i}-j: 0 \leq j \leq t-1\right\}$.

$(C 2)$ Let $\lambda^{+}=\left(\bar{\lambda}_{t \text {-core }} ;\left(\bar{\lambda}^{0}\right)^{+}, \lambda^{1}, \ldots, \lambda^{t^{\prime}}\right)$ be a doubled distinct partition such that $\left(\bar{\lambda}^{0}\right)^{+}=\bar{\lambda}^{0} \cup\left\{\square_{0}\right\}$. We have

$\mathcal{C}\left(\lambda^{+}\right) \backslash \mathcal{C}(\lambda)=\left\{t c_{\square_{0}}+b_{0}-j: 0 \leq j \leq t-1\right\} \cup\left\{t\left(1-c_{\square_{0}}\right)+b_{0}-j: 0 \leq j \leq t-1\right\}$. 
Hence, $\sum_{c \in \mathcal{C}(\lambda)} c^{r}$ is $\mu$-admissible for any nonnegative integer $r$.

On the other hand, we obtain the following results by Lemma 4.6.

$(H 1)$ Let $1 \leq i \leq t^{\prime}$ and $\lambda^{+}=\left(\bar{\lambda}_{t \text {-core }} ; \bar{\lambda}^{0}, \lambda^{1}, \ldots, \lambda^{i-1},\left(\lambda^{i}\right)^{+}, \lambda^{i+1}, \ldots, \lambda^{t^{\prime}}\right)$ be a doubled distinct partition such that $\left(\lambda^{i}\right)^{+}=\lambda^{i} \cup\left\{\square_{i}\right\}$. We have

$$
\begin{aligned}
& \sum_{\square \in \lambda^{+}} h_{\square}^{2 r}-\sum_{\square \in \lambda} h_{\square}^{2 r} \\
& =t^{2 r}+\left(t c_{\square_{i}}+b_{i}-b_{0}\right)^{2 r} \\
& +\sum_{j=1}^{t-1}\left(\sum_{0 \leq s \leq m_{j}}\left(t c_{\square_{i}}+b_{i}-t x_{j, s}-b_{j}\right)^{2 r}-\sum_{1 \leq s \leq m_{j}}\left(t c_{\square_{i}}+b_{i}-t y_{j, s}-b_{j}\right)^{2 r}\right) \\
& +\sum_{1 \leq s \leq m_{j}}\left(t c_{\square_{i}}+b_{i}-t x_{0, s}-b_{0}\right)^{2 r}-\sum_{1 \leq s \leq m_{j}}\left(t c_{\square_{i}}+b_{i}-t y_{0, s}-b_{0}\right)^{2 r} \\
& +\sum_{1 \leq s \leq m_{j}}\left(t c_{\square_{i}}+b_{i}-t\left(1-x_{0, s}\right)-b_{0}\right)^{2 r}-\sum_{1 \leq s \leq m_{j}}\left(t c_{\square_{i}}+b_{i}-t\left(1-y_{0, s}\right)-b_{0}\right)^{2 r} \\
& +t^{2 r}+\left(-t c_{\square_{i}}+b_{t-i}-b_{0}\right)^{2 r} \\
& +\sum_{j=1}^{t-1}\left(\sum_{0 \leq s \leq m_{j}}\left(-t c_{\square_{i}}+b_{t-i}-t x_{j, s}-b_{j}\right)^{2 r}-\sum_{1 \leq s \leq m_{j}}\left(-t c_{\square_{i}}+b_{t-i}-t y_{j, s}-b_{j}\right)^{2 r}\right) \\
& +\sum_{1 \leq s \leq m_{j}}\left(-t c_{\square_{i}}+b_{t-i}-t x_{0, s}-b_{0}\right)^{2 r}-\sum_{1 \leq s \leq m_{j}}\left(-t c_{\square_{i}}+b_{t-i}-t y_{0, s}-b_{0}\right)^{2 r} \\
& +\sum_{1 \leq s \leq m_{j}}\left(-t c_{\square_{i}}+b_{t-i}-t\left(1-x_{0, s}\right)-b_{0}\right)^{2 r} \\
& -\sum_{1 \leq s \leq m_{j}}\left(-t c_{\square_{i}}+b_{t-i}-t\left(1-y_{0, s}\right)-b_{0}\right)^{2 r}+\left(-t c_{\square_{i}}+b_{t-i}-t\left(c_{\square_{i}}+1\right)-b_{0}\right)^{2 r} \\
& +\left(-t c_{\square_{i}}+b_{t-i}-t\left(c_{\square_{i}}-1\right)-b_{0}\right)^{2 r}-2\left(-t c_{\square_{i}}+b_{t-i}-t c_{\square_{i}}-b_{0}\right)^{2 r} .
\end{aligned}
$$

(H2) Let $\lambda^{+}=\left(\bar{\lambda}_{t \text {-core }} ;\left(\bar{\lambda}^{0}\right)^{+}, \lambda^{1}, \ldots, \lambda^{t^{\prime}}\right)$ be a doubled distinct partition such that $\left(\bar{\lambda}^{0}\right)^{+}=\bar{\lambda}^{0} \cup\left\{\square_{0}\right\}$. We have

$$
\begin{aligned}
& \sum_{\square \in \lambda^{+}} h_{\square}^{2 r}-\sum_{\square \in \lambda} h_{\square}^{2 r} \\
& =t^{2 r}+\left(t c_{\square_{0}}\right)^{2 r} \\
& +\sum_{j=1}^{t-1}\left(\sum_{0 \leq s \leq m_{j}}\left(t c_{\square_{0}}+b_{0}-t x_{j, s}-b_{j}\right)^{2 r}-\sum_{1 \leq s \leq m_{j}}\left(t c_{\square_{0}}+b_{0}-t y_{j, s}-b_{j}\right)^{2 r}\right) \\
& +\sum_{1 \leq s \leq m_{j}}\left(t c_{\square_{0}}-t x_{0, s}\right)^{2 r}-\sum_{1 \leq s \leq m_{j}}\left(t c_{\square_{0}}-t y_{0, s}\right)^{2 r} \\
& +\sum_{1 \leq s \leq m_{j}}\left(t c_{\square_{0}}-t\left(1-x_{0, s}\right)\right)^{2 r}-\sum_{1 \leq s \leq m_{j}}\left(t c_{\square_{0}}-t\left(1-y_{0, s}\right)\right)^{2 r} \\
& +t^{2 r}+\left(t-t c_{\square_{0}}\right)^{2 r}
\end{aligned}
$$




$$
\begin{aligned}
& +\sum_{j=1}^{t-1}\left(\sum_{0 \leq s \leq m_{j}}\left(t-t c_{\square_{0}}+b_{0}-t x_{j, s}-b_{j}\right)^{2 r}-\sum_{1 \leq s \leq m_{j}}\left(t-t c_{\square_{0}}+b_{0}-t y_{j, s}-b_{j}\right)^{2 r}\right) \\
& +\sum_{1 \leq s \leq m_{j}}\left(t-t c_{\square_{0}}-t x_{0, s}\right)^{2 r}-\sum_{1 \leq s \leq m_{j}}\left(t-t c_{\square_{0}}-t y_{0, s}\right)^{2 r} \\
& +\sum_{1 \leq s \leq m_{j}}\left(t-t c_{\square_{0}}-t\left(1-x_{0, s}\right)\right)^{2 r}-\sum_{1 \leq s \leq m_{j}}\left(t-t c_{\square_{0}}-t\left(1-y_{0, s}\right)\right)^{2 r} \\
& +\left(t-t c_{\square_{0}}-t\left(c_{\square_{0}}+1\right)\right)^{2 r}+\left(t-t c_{\square_{0}}-t\left(c_{\square_{0}}-1\right)\right)^{2 r} \\
& -2\left(t-t c_{\square_{0}}-t c_{\square_{0}}\right)^{2 r} .
\end{aligned}
$$

Hence, $\sum_{h \in \mathcal{H}(\lambda)} h^{2 r}$ is $\mu$-admissible for any nonnegative integer $r$.

4.3. Main results for doubled distinct partitions. To prove the doubled distinct case of Theorem 1.4, we establish the following more general result.

Theorem 4.7. Let $\left(\nu^{0}, \nu^{1}, \ldots, \nu^{t^{\prime}}\right)$ be a $\left(t^{\prime}+1\right)$-tuple of usual partitions, and $\alpha$ be a t-core doubled distinct partition. Suppose that $g_{1}, g_{2}, \ldots, g_{v}$ are $\alpha$-admissible functions of doubled distinct partitions. Then, there exists some $r \in \mathbb{N}$ such that

$$
\left(D_{t}^{\mathcal{D} \mathcal{D}}\right)^{r}\left(G_{\lambda}^{\mathcal{D} \mathcal{D}} \prod_{u=1}^{v} g_{u}(\lambda) \Phi^{\nu^{0}}\left(\bar{\lambda}^{0}\right) \prod_{i=1}^{t^{\prime}} \Psi^{\nu^{i}}\left(\lambda^{i}\right)\right)=0
$$

for every doubled distinct partition $\lambda$ with $\lambda_{t \text {-core }}=\alpha$. Furthermore, let $\mu$ be a given doubled distinct partition. By Theorem 4.3,

$$
\sum_{\substack{\lambda \in \mathcal{D} \mathcal{D}, \lambda \geq t \mu \\|\lambda / \mu|=2 n t}} F_{\lambda / \mu}^{\mathcal{D D}} G_{\lambda}^{\mathcal{D D}} \prod_{u=1}^{v} g_{u}(\lambda)
$$

is a polynomial in $n$. Moreover, if

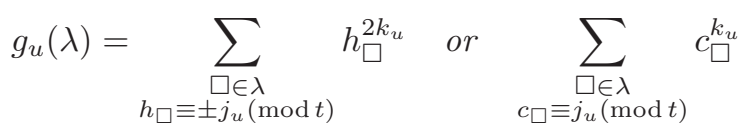

for each $1 \leq u \leq v$ where $j_{u}, k_{u} \in \mathbb{N}$, then (4.9) is also a polynomial of $t$.

Proof. We will prove this claim by induction. Let

$$
\begin{aligned}
A & =\prod_{u=1}^{v} g_{u}(\lambda), \quad B=\prod_{i=1}^{t^{\prime}} \Psi^{\nu^{i}}\left(\lambda^{i}\right), \quad C=\Phi^{\nu^{0}}\left(\bar{\lambda}^{0}\right), \\
\Delta A & =\prod_{u=1}^{v} g_{u}(\rho)-\prod_{u=1}^{v} g_{u}(\lambda)=\sum_{(*)} \prod_{s \in U} g_{s}(\lambda) \prod_{s^{\prime} \in V}\left(g_{s^{\prime}}(\rho)-g_{s^{\prime}}(\lambda)\right), \\
\Delta B & =\prod_{i=1}^{t^{\prime}} \Psi^{\nu^{i}}\left(\rho^{i}\right)-\prod_{i=1}^{t^{\prime}} \Psi^{\nu^{i}}\left(\lambda^{i}\right) \\
& =\sum_{(* *)} \prod_{s \in U} \Psi^{\nu^{s}}\left(\lambda^{i}\right) \prod_{s^{\prime} \in V}\left(\Psi^{\nu^{s^{\prime}}}\left(\rho^{i}\right)-\Psi^{\nu^{s^{\prime}}}\left(\lambda^{i}\right)\right), \\
\Delta C & =\Phi^{\nu^{0}}\left(\bar{\rho}^{0}\right)-\Phi^{\nu^{0}}\left(\bar{\lambda}^{0}\right),
\end{aligned}
$$


GUO-NIU HAN AND HUAN XIONG*

where the sum $(*)$ (resp. $(* *))$ ranges over all pairs $(U, V)$ of positive integer sets such that $U \cup V=\{1,2, \ldots, v\}$ (resp. $U \cup V=\left\{1,2, \ldots, t^{\prime}\right\}$ ), $U \cap V=\emptyset$ and $V \neq \emptyset$. We have

$$
\begin{aligned}
& D_{t}^{\mathcal{D D}}\left(G_{\lambda}^{\mathcal{D} \mathcal{D}} \prod_{u=1}^{v} g_{u}(\lambda) \Phi^{\nu^{0}}\left(\bar{\lambda}^{0}\right) \prod_{i=1}^{t^{\prime}} \Psi^{\nu^{i}}\left(\lambda^{i}\right)\right) \\
= & G_{\lambda}^{\mathcal{D D}} \sum_{\substack{\rho \geq t \lambda \\
|\rho / \lambda|=2 t}} \frac{G_{\rho}^{\mathcal{D} \mathcal{D}}}{G_{\lambda}^{\mathcal{D} \mathcal{D}}}\left(\prod_{u=1}^{v} g_{u}(\rho) \Phi^{\nu^{0}}\left(\bar{\rho}^{0}\right) \prod_{i=1}^{t^{\prime}} \Psi^{\nu^{i}}\left(\rho^{i}\right)\right. \\
& \left.-\prod_{u=1}^{v} g_{u}(\lambda) \Phi^{\nu^{0}}\left(\bar{\lambda}^{0}\right) \prod_{i=1}^{t^{\prime}} \Psi^{\nu^{i}}\left(\lambda^{i}\right)\right) \\
= & G_{\lambda}^{\mathcal{D D}} \sum_{\substack{\rho \geq t \lambda \\
|\rho / \lambda|=2 t}} \frac{G_{\rho}^{\mathcal{D} \mathcal{D}}}{G_{\lambda}^{\mathcal{D D}}}(\Delta A \cdot B \cdot C+A \cdot \Delta B \cdot C+A \cdot B \cdot \Delta C \\
+ & A \cdot \Delta B \cdot \Delta C+\Delta A \cdot B \cdot \Delta C+\Delta A \cdot \Delta B \cdot C+\Delta A \cdot \Delta B \cdot \Delta C) .
\end{aligned}
$$

For the first term in the above summation, we obtain

$$
\begin{aligned}
& G_{\lambda}^{\mathcal{D D}} \sum_{\substack{\rho \geq t \lambda \\
|\rho / \lambda|=2 t}} \frac{G_{\rho}^{\mathcal{D D}}}{G_{\lambda}^{\mathcal{D D}}}(\Delta A \cdot B \cdot C) \\
& =\frac{1}{t} G_{\lambda}^{\mathcal{D} \mathcal{D}} \Phi^{\nu^{0}}\left(\bar{\lambda}^{0}\right) \prod_{i=1}^{t^{\prime}} \Psi^{\nu^{i}}\left(\lambda^{i}\right) \sum_{0 \leq i \leq m_{0}} \frac{\left.\prod_{\substack{1 \leq j \leq m_{0} \\
0 \leq j \leq m_{0} \\
j \neq i}}\left(\left(\begin{array}{c}
x_{0, i} \\
2
\end{array}\right)-\left(\begin{array}{c}
y_{0, j}, i \\
2
\end{array}\right)\right)-\left(\begin{array}{c}
x_{0, j} \\
2
\end{array}\right)\right)}{\left.\prod_{\substack{x_{0} \\
j}}\right)} \\
& \times \sum_{(*)} \prod_{s \in U} g_{s}(\lambda) \prod_{s^{\prime} \in V}\left(g_{s^{\prime}}\left(\left(\bar{\mu} ;\left(\bar{\lambda}^{0}\right)^{i+}, \lambda^{1}, \ldots, \lambda^{t^{\prime}}\right)\right)-g_{s^{\prime}}(\lambda)\right) \\
& +\frac{2}{t} G_{\lambda}^{\mathcal{D} \mathcal{D}} \Phi^{\nu^{0}}\left(\bar{\lambda}^{0}\right) \prod_{i=1}^{t^{\prime}} \Psi^{\nu^{i}}\left(\lambda^{i}\right) \sum_{k=1}^{t^{\prime}} \sum_{0 \leq i \leq m_{k}} \frac{\prod_{\substack{1 \leq j \leq m_{k} \\
0 \leq j \leq m_{k} \\
j \neq i}}\left(x_{k, i}-y_{k, j}\right)}{\left.\prod_{k, i}-x_{k, j}\right)} \\
& \times \sum_{(*)} \prod_{s \in U} g_{s}(\lambda) \prod_{s^{\prime} \in V}\left(g_{s^{\prime}}\left(\left(\bar{\mu} ; \bar{\lambda}^{0}, \lambda^{1}, \ldots,\left(\lambda^{k}\right)^{i+}, \ldots, \lambda^{t^{\prime}}\right)\right)-g_{s^{\prime}}(\lambda)\right)
\end{aligned}
$$

where $\mathcal{C}\left(\left(\bar{\lambda}^{0}\right)^{i+}\right) \backslash \mathcal{C}\left(\bar{\lambda}^{0}\right)=x_{0, i}$ and $\mathcal{C}\left(\left(\lambda^{k}\right)^{i+}\right) \backslash \mathcal{C}\left(\lambda^{k}\right)=x_{k, i}$. Since $g_{1}, g_{2}, \ldots, g_{v}$ are $\alpha$-admissible functions and thanks to Lemma 2.4,

$$
G_{\lambda}^{\mathcal{D D}} \sum_{\substack{\rho \geq t \lambda \\|\rho / \lambda|=2 t}} \frac{G_{\rho}^{\mathcal{D D}}}{G_{\lambda}^{\mathcal{D D}}}(\Delta A \cdot B \cdot C)
$$

could be written as a linear combination of some

$$
G_{\lambda}^{\mathcal{D D}} \prod_{\underline{u}=1}^{\underline{v}} g_{\underline{u}}^{\prime}(\lambda) \Phi^{\underline{\nu}^{0}}\left(\bar{\lambda}^{0}\right) \prod_{i=1}^{t^{\prime}} \Psi^{\underline{\nu}^{i}}\left(\lambda^{i}\right)
$$


for some $\alpha$-admissible functions $g_{\underline{u}}^{\prime}$, where either $\underline{v}<v$, or $\underline{v}=v$ and simultaneously

$$
2\left|\underline{\nu}^{0}\right|+\sum_{i=1}^{t^{\prime}}\left|\underline{\nu}^{i}\right| \leq 2\left|\nu^{0}\right|+\sum_{i=1}^{t^{\prime}}\left|\nu^{i}\right|-2 .
$$

On the other hand, we have similar results for other six terms by Lemmas 3.1, 2.4 and Theorem 2.2. Thus, the polynomiality of (4.9) in $n$ is proved by induction on $\left(v, 2\left|\nu^{0}\right|+\sum_{i=1}^{t^{\prime}}\left|\nu^{i}\right|\right)$. Also, notice that $b_{i} \equiv i(\bmod t)$, then by $(\mathrm{C} 1),(\mathrm{C} 2),(\mathrm{H} 1)$, (H2) in the proof of Lemma 4.4 we have, for each $j, k \in \mathbb{N}$,

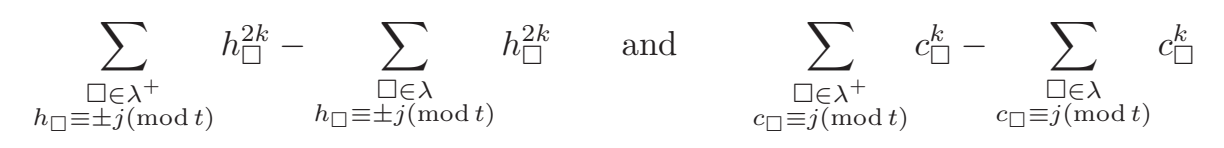

can be written as some polynomials of $t$ with degrees at most $2 k+1$ and $k+1$ respectively, whose constant terms are 0 , and other coefficients are some admissible functions of $\lambda$ (independent of $t$ ). Moreover, if

$$
g_{u}(\lambda)=\sum_{\substack{\square \in \lambda \\ h_{\square} \equiv \pm j_{u}(\bmod t)}} h_{\square}^{2 k_{u}} \text { or } \sum_{\substack{\square \in \lambda \\ c_{\square} \equiv j_{u}(\bmod t)}} c_{\square}^{k_{u}}
$$

for each $1 \leq u \leq v$ where $j_{u}, k_{u} \in \mathbb{N}$, by previous analysis on each term of (4.10) and induction,

$$
\left(D_{t}^{\mathcal{D D}}\right)^{r}\left(G_{\lambda}^{\mathcal{D D}} \prod_{u=1}^{v} g_{u}(\lambda)\right)
$$

is a polynomial of $t$, when $\lambda$ is fixed. Therefore, again by Theorem 4.3, we know (4.9) is also a polynomial in $t$.

As an application of Theorem 4.7, we derive the doubled distinct partition case of Theorem 1.4 from Lemma 4.4 by letting $\mu=\emptyset$. Actually, by a similar but more precise argument as in the proof of Lemma 4.4, we can show that

$$
\sum_{\substack{\square \in \lambda \\ h_{\square} \equiv \pm j(\bmod t)}} h_{\square}^{2 r} \text { and } \sum_{\substack{\square \in \lambda \\ c_{\square} \equiv j(\bmod t)}} c_{\square}^{r}
$$

are $\mu$-admissible for any $t$-core doubled distinct partition $\mu$, any nonnegative integer $r$, and $0 \leq j \leq t-1$. By the proof of Theorem 4.7 we derive the following result.

Corollary 4.8. Let $u^{\prime}, v^{\prime}, j_{u}, j_{v}^{\prime}, k_{u}, k_{v}^{\prime}$ be nonnegative integers and $\alpha$ be a given $t$-core doubled distinct partition. Then, there exists some $r \in \mathbb{N}$ such that

$$
\left(D_{t}^{\mathcal{D D}}\right)^{r}\left(G_{\lambda}^{\mathcal{D} \mathcal{D}}\left(\prod_{u=1}^{u^{\prime}} \sum_{\substack{\square \in \lambda \\ h_{\square} \equiv \pm j_{u}(\bmod t)}} h_{\square}^{2 k_{u}}\right)\left(\prod_{v=1}^{v^{\prime}} \sum_{\substack{\square \in \lambda \\ c_{\square} \equiv j_{v}^{\prime}(\bmod t)}} c_{\square}^{k_{v}^{\prime}}\right)\right)=0
$$

for every doubled distinct partition $\lambda$ with $\lambda_{t \text {-core }}=\alpha$. Moreover, let $\mu$ be a given doubled distinct partition. Then

$$
\sum_{\substack{\lambda \in \mathcal{D} \mathcal{D}, \lambda \geq \geq_{t} \mu \\|\lambda / \mu|=2 n t}} F_{\lambda / \mu}^{\mathcal{D D}} G_{\lambda}^{\mathcal{D D}}\left(\prod_{u=1}^{u^{\prime}} \sum_{\substack{\square \in \lambda \\ h_{\square} \equiv \pm j_{u}(\bmod t)}} h_{\square}^{2 k_{u}}\right)\left(\prod_{v=1}^{v^{\prime}} \sum_{\substack{\square \in \lambda \\ c_{\square} \equiv j_{v}^{\prime}(\bmod t)}} c_{\square}^{k_{v}^{\prime}}\right)
$$


is a polynomial in $n$ of degree at most $\sum_{u=1}^{u^{\prime}}\left(k_{u}+1\right)+\sum_{v=1}^{v^{\prime}} \frac{k_{v}^{\prime}+2}{2}$, and a polynomial in $t$ of degree at most $\sum_{u=1}^{u^{\prime}}\left(2 k_{u}+1\right)+\sum_{v=1}^{v^{\prime}}\left(k_{v}^{\prime}+1\right)$.

\section{Polynomiality for Self-CONJugate Partitions}

In this section we always set that $t=2 t^{\prime}$ is an even positive integer. The set of all $t$-core self-conjugate partitions is denoted by $\mathcal{S C}_{t \text {-core }}$. Let $\lambda$ be a self-conjugate partition. By [6], the Littlewood decomposition maps $\lambda$ to

$$
\left(\lambda_{t \text {-core }} ; \lambda^{0}, \lambda^{1}, \ldots, \lambda^{t-1}\right) \in \mathcal{S C}_{t \text {-core }} \times \mathcal{P}^{2 t^{\prime}}
$$

where $\lambda^{i}$ is the conjugate partition of $\lambda^{t-1-i}$ for $0 \leq i \leq t^{\prime}-1$. For convenience and similarly to the case of doubled distinct partitions, we always write

$$
\lambda=\left(\lambda_{t \text {-core }} ; \lambda^{0}, \ldots, \lambda^{t^{\prime}-1}\right) .
$$

Let $\lambda=\left(\lambda_{t \text {-core }} ; \lambda^{0}, \ldots, \lambda^{t^{\prime}-1}\right)$ and $\mu=\left(\mu_{t \text {-core }} ; \mu^{0}, \ldots, \mu^{t^{\prime}-1}\right)$ be two self-conjugate partitions. If $\lambda_{t \text {-core }}=\mu_{t \text {-core }}$ and $\lambda^{i} \supset \mu^{i}$ for $0 \leq i \leq t^{\prime}-1$, we write $\lambda \geq_{t} \mu$ and define

$$
F_{\mu / \mu}^{\mathcal{S C}}:=1 \quad \text { and } \quad F_{\lambda / \mu}^{\mathcal{S C}}:=\sum_{\substack{\lambda \geq t \lambda^{-} \geq_{t} \mu \\\left|\lambda / \lambda^{-}\right|=2 t}} F_{\lambda^{-} / \mu}^{\mathcal{S C}} \quad(\text { for } \lambda \neq \mu) .
$$

Then $F_{\lambda / \mu}^{\mathcal{S C}}$ is the number of vectors $\left(P_{0}, P_{1}, \ldots, P_{t^{\prime}-1}\right)$ such that

(1) $P_{i}\left(0 \leq i \leq t^{\prime}-1\right)$ is a skew Young tableau of shape $\lambda^{i} / \mu^{i}$,

(2) the union of entries in $P_{0}, P_{1}, \ldots, P_{t^{\prime}}$ are $\left\{1,2, \ldots, n=\sum_{i=0}^{t^{\prime}-1}\left|\lambda^{i} / \mu^{i}\right|\right\}$. Hence,

$$
F_{\lambda / \mu}^{\mathcal{S C}}=\left(\begin{array}{c}
\sum_{i=0}^{t^{\prime}-1}\left|\lambda^{i} / \mu^{i}\right| \\
\left|\lambda^{0} / \mu^{0}\right|, \ldots,\left|\lambda^{t^{\prime}} / \mu^{t^{\prime}}\right|
\end{array}\right) \prod_{i=0}^{t^{\prime}-1} f_{\lambda^{i} / \mu^{i}}
$$

We set

$$
F_{\lambda}^{\mathcal{S C}}:=F_{\lambda / \lambda_{t-\text { core }}}^{\mathcal{S C}}=\left(\begin{array}{c}
\sum_{i=0}^{t^{\prime}-1}\left|\lambda^{i}\right| \\
\left|\lambda^{0}\right|, \ldots,\left|\lambda^{t^{\prime}-1}\right|
\end{array}\right) \prod_{i=0}^{t^{\prime}-1} f_{\lambda^{i}}=\frac{n !}{\prod_{i=0}^{t^{\prime}-1} H\left(\lambda^{i}\right)}
$$

and

$$
G_{\lambda}^{\mathcal{S C}}:=\frac{2^{n}}{t^{n} \prod_{i=0}^{t^{\prime}-1} H\left(\lambda^{i}\right)}=\frac{2^{n} F_{\lambda}^{\mathcal{S C}}}{t^{n} n !} .
$$

Let $g: \mathcal{S C} \rightarrow \mathbb{R}$ be a function of self-conjugate partitions and $\lambda$ be a selfconjugate partition. The $t$-difference operator $D_{t}^{\mathcal{S C}}$ for self-conjugate partitions is defined by

$$
D_{t}^{\mathcal{S C}} g(\lambda)=\sum_{\substack{\lambda^{+} \geq t \lambda \\\left|\lambda^{+} / \lambda\right|=2 t}} g\left(\lambda^{+}\right)-g(\lambda) .
$$

The higher-order $t$-difference operators $D_{t}^{k}$ are defined by induction:

$$
\left(D_{t}^{\mathcal{S C}}\right)^{0} g:=g \quad \text { and } \quad\left(D_{t}^{\mathcal{S C}}\right)^{k} g:=D_{t}^{\mathcal{S C}}\left(\left(D_{t}^{\mathcal{S C}}\right)^{k-1} g\right) \quad(k \geq 1) .
$$


Lemma 5.1. Suppose that $\lambda$ is a self-conjugate partition. Then $D_{t}^{\mathcal{S C}}\left(G_{\lambda}^{\mathcal{S C}}\right)=0$. In other words,

$$
G_{\lambda}^{\mathcal{S C}}=\sum_{\substack{\lambda^{+} \geq t^{\lambda} \\\left|\lambda^{+} / \lambda\right|=2 t}} G_{\lambda^{+}}^{\mathcal{S C}}
$$

Proof. Write $\lambda=\left(\lambda_{t \text {-core }} ; \lambda^{0}, \ldots, \lambda^{t^{\prime}-1}\right)$. For $0 \leq i \leq t^{\prime}-1$, we obtain

$$
\sum_{\left|\left(\lambda^{i}\right)^{+} / \lambda^{i}\right|=1} \frac{G_{\left(\lambda t \text {-core } ; \lambda^{0}, \ldots, \lambda^{i-1},\left(\lambda^{i}\right)^{+}, \lambda^{i+1}, \ldots, \lambda^{t^{\prime}-1}\right)}^{\mathcal{S C}}}{G_{\lambda}^{\mathcal{S C}}}=\sum_{\left|\left(\lambda^{i}\right)^{+} / \lambda^{i}\right|=1} \frac{2 H\left(\lambda^{i}\right)}{t H\left(\left(\lambda^{i}\right)^{+}\right)}=\frac{2}{t}
$$

by Lemma 2.2 in [11]. Summing the above equalities we prove (5.4).

By analogy with the results on doubled distinct partitions, we have the following theorems for self-conjugate partitions. Their proofs are omitted.

Lemma 5.2. Suppose that $\mu$ is a given self-conjugate partition and $g: \mathcal{S C} \rightarrow \mathbb{R}$ is a function of self-conjugate partitions. For every nonnegative integer n, let

$$
P(\mu, g ; n):=\sum_{\substack{\lambda \in \mathcal{S C}, \lambda \geq t \mu \\|\lambda / \mu|=2 n t}} F_{\lambda / \mu}^{\mathcal{S C}} g(\lambda)
$$

Then

$$
P(\mu, g ; n+1)-P(\mu, g ; n)=P\left(\mu, D_{t}^{\mathcal{S C}} g ; n\right) .
$$

Example 5.1. Let $g(\lambda)=G_{\lambda}^{\mathcal{S C}}$. Then $D_{t}^{\mathcal{S C}} g(\lambda)=0$ by Lemma 5.1, which means that

$$
\sum_{\substack{\lambda \in \mathcal{S C}, \lambda \geq t \mu \\|\lambda / \mu|=2 n t}} F_{\lambda / \mu}^{\mathcal{S C}} G_{\lambda}^{\mathcal{S C}}=G_{\mu}^{\mathcal{S C}}
$$

When $\mu=\emptyset$, the above identity becomes

$$
\sum_{\substack{\lambda \in \mathcal{S C},|\lambda|=2 n t \\ \lambda t-\text { core }=\emptyset}} \frac{(2 t)^{n} n !}{\prod_{h \in \mathcal{H}_{t}(\lambda)} h}=1
$$

Theorem 5.3. Let $g: \mathcal{S C} \rightarrow \mathbb{R}$ be a function of self-conjugate partitions and $\mu$ be a given self-conjugate partition. Then,

$$
P(\mu, g ; n)=\sum_{\substack{\lambda \in \mathcal{S C}, \lambda \geq t \mu \\
|\lambda / \mu|=2 n t}} F_{\lambda / \mu}^{\mathcal{S C}} g(\lambda)=\sum_{k=0}^{n}\left(\begin{array}{l}
n \\
k
\end{array}\right)\left(D_{t}^{\mathcal{S C}}\right)^{k} g(\mu)
$$

and

$$
\left(D_{t}^{\mathcal{S C}}\right)^{n} g(\mu)=\sum_{k=0}^{n}(-1)^{n+k}\left(\begin{array}{l}
n \\
k
\end{array}\right) P(\mu, g ; k) .
$$

In particular, if there exists some positive integer $r$ such that $\left(D_{t}^{\mathcal{S C}}\right)^{r} g(\lambda)=0$ for every self-conjugate partition $\lambda \geq_{t} \mu$, then $P(\mu, g ; n)$ is a polynomial in $n$ of degree at most $r-1$. 
Theorem 5.4. Let $t=2 t^{\prime}$ be a given integer, $\alpha$ be a given $t$-core self-conjugate partition, and $u^{\prime}, v^{\prime}, j_{u}, j^{\prime}{ }_{v}, k_{u}, k_{v}^{\prime}$ be nonnegative integers. Then there exists some $r \in \mathbb{N}$ such that

$$
\left(D_{t}^{\mathcal{S C}}\right)^{r}\left(G_{\lambda}\left(\prod_{u=1}^{u^{\prime}} \sum_{\substack{\square \in \lambda \\ h_{\square} \equiv \pm j_{u}(\bmod t)}} h_{\square}^{2 k_{u}}\right)\left(\prod_{v=1}^{v^{\prime}} \sum_{\substack{\square \in \lambda \\ c \square \equiv j_{v}^{\prime}(\bmod t)}} c_{\square}^{k_{v}^{\prime}}\right)\right)=0
$$

for every self-conjugate partition $\lambda$ with $\lambda_{t \text {-core }}=\alpha$. Furthermore, let $\mu$ be a given self-conjugate partition. Then by Theorem 5.3, we have

$$
\sum_{\substack{\lambda \in \mathcal{S C}, \lambda \geq t \mu \\|\lambda / \mu|=2 n t}} F_{\lambda / \mu}^{\mathcal{S C}} G_{\lambda}^{\mathcal{S C}}\left(\prod_{u=1}^{u^{\prime}} \sum_{\substack{\square \in \lambda \\ h_{\square} \equiv \pm j_{u}(\bmod t)}} h_{\square}^{2 k_{u}}\right)\left(\prod_{v=1}^{v^{\prime}} \sum_{\substack{\square \in \lambda \\ c_{\square} \equiv j_{v}^{\prime}(\bmod t)}} c_{\square}^{k_{v}^{\prime}}\right)
$$

is a polynomial in $n$ and $t$. The degrees of this polynomial for $n$ and $t$ are at most $\sum_{u=1}^{u^{\prime}}\left(k_{u}+1\right)+\sum_{v=1}^{v^{\prime}} \frac{k_{v}^{\prime}+2}{2}$ and $\sum_{u=1}^{u^{\prime}}\left(2 k_{u}+1\right)+\sum_{v=1}^{v^{\prime}}\left(k_{v}^{\prime}+1\right)$, respectively.

By letting $\mu=\emptyset$ in Theorem 5.4 we derive the self-conjugate partition case of Theorem 1.4.

\section{Square CASES For Doubled Distinct AND SELF-CONJUGate Partitions}

As described in Corollary 1.5, the polynomials mentioned in Corollary 4.8 and Theorem 5.4 have explicit expressions for square cases.

Proof of Corollary 1.5. (1) When $\lambda$ is a doubled distinct partition with $|\lambda|=2 n t$ ( $t$ odd) and $\lambda_{t \text {-core }}=\emptyset$. By the proof of Lemma 4.4 we obtain

$$
\begin{aligned}
& \frac{1}{G_{\lambda}^{\mathcal{D D}}} D_{t}^{\mathcal{D D}}\left(G_{\lambda}^{\mathcal{D D}}\left(\sum_{\square \in \lambda} c_{\square}^{2}\right)\right)=\frac{1}{t} \sum_{0 \leq i \leq m_{0}} \frac{\prod_{\substack{1 \leq j \leq m_{0} \\
0 \leq j \leq m_{0} \\
j \neq i}}\left(\left(\begin{array}{c}
x_{0, i} \\
2
\end{array}\right)-\left(\begin{array}{c}
y_{0, j} \\
2
\end{array}\right)\right)}{\left.\left.\prod_{2}\right)-\left(\begin{array}{c}
x_{0, j} \\
2
\end{array}\right)\right)} \\
& \times \sum_{j=0}^{t-1}\left(\left(t x_{0, i}-j\right)^{2}+\left(t-t x_{0, i}-j\right)^{2}\right) \\
& +\frac{2}{t} \sum_{1 \leq k \leq t^{\prime}} \sum_{0 \leq i \leq m_{k}} \frac{\prod_{\substack{1 \leq j \leq m_{k} \\
0 \leq j \leq m_{k} \\
j \neq i}}\left(x_{k, i}-y_{k, j}\right)}{\left.\prod_{k, i}-x_{k, j}\right)} \\
& \times \sum_{j=0}^{t-1}\left(\left(t x_{k, i}+k-j\right)^{2}+\left(-t x_{k, i}+t-k-j\right)^{2}\right) \\
& =\frac{1}{t} \sum_{0 \leq i \leq m_{0}} \frac{\prod_{\substack{1 \leq j \leq m_{0} \\
0 \leq j \leq m_{0} \\
j \neq i}}\left(\left(\begin{array}{c}
x_{0, i} \\
2
\end{array}\right)-\left(\begin{array}{c}
y_{0, j} \\
2
\end{array}\right)\right)}{\left.\left.\prod_{0, i}\right)-\left(\begin{array}{c}
x_{0, j} \\
2
\end{array}\right)\right)} \\
& \times\left(4 t^{3}\left(\begin{array}{c}
x_{0, i} \\
2
\end{array}\right)+t^{3}-t^{2}(t-1)+\frac{(t-1) t(2 t-1)}{3}\right)
\end{aligned}
$$




$$
\begin{aligned}
& +\frac{2}{t} \sum_{1 \leq k \leq t^{\prime}} \sum_{0 \leq i \leq m_{k}} \frac{\prod_{\substack{1 \leq j \leq m_{k} \\
0 \leq j \leq m_{k} \\
j \neq i}}\left(x_{k, i}-y_{k, j}\right)}{\left.\prod_{k, i}-x_{k, j}\right)} \\
& \quad \times\left(2 t^{3} x_{k, i}^{2}+\sum_{j=0}^{t-1}(k-j)^{2}+\sum_{j=0}^{t-1}(t-k-j)^{2}\right) \\
& =2 t|\lambda|+\frac{t\left(t^{2}+2\right)}{3},
\end{aligned}
$$

therefore

$$
\frac{1}{G_{\lambda}^{\mathcal{D D}}}\left(D_{t}^{\mathcal{D D}}\right)^{2}\left(G_{\lambda}^{\mathcal{D D}}\left(\sum_{\square \in \lambda} c_{\square}^{2}\right)\right)=4 t^{2}
$$

and

$$
\frac{1}{G_{\lambda}^{\mathcal{D D}}}\left(D_{t}^{\mathcal{D D}}\right)^{3}\left(G_{\lambda}^{\mathcal{D D}}\left(\sum_{\square \in \lambda} c_{\square}^{2}\right)\right)=0 .
$$

(2) When $\lambda$ is a self-conjugate partition with $|\lambda|=2 n t$ ( $t$ even) and $\lambda_{t \text {-core }}=\emptyset$. Similarly as in (1) we have

$$
\begin{gathered}
\frac{1}{G_{\lambda}^{\mathcal{S C}}} D_{t}^{\mathcal{S C}}\left(G_{\lambda}^{\mathcal{S C}}\left(\sum_{\square \in \lambda} c_{\square}^{2}\right)\right)=2 t|\lambda|+\frac{t\left(t^{2}-1\right)}{3}, \\
\frac{1}{G_{\lambda}^{\mathcal{S C}}}\left(D_{t}^{\mathcal{S C}}\right)^{2}\left(G_{\lambda}^{\mathcal{S C}}\left(\sum_{\square \in \lambda} c_{\square}^{2}\right)\right)=4 t^{2},
\end{gathered}
$$

and

$$
\frac{1}{G_{\lambda}^{\mathcal{S C}}}\left(D_{t}^{\mathcal{S C}}\right)^{3}\left(G_{\lambda}^{\mathcal{S C}}\left(\sum_{\square \in \lambda} c_{\square}^{2}\right)\right)=0 .
$$

Then identities (1.8) and (1.9) follows from Theorems 4.3 and 5.3. Notice that $\sum_{\square \in \lambda} h_{\square}^{2}-\sum_{\square \in \lambda} c_{\square}^{2}=|\lambda|^{2}$ (see [17]). Identities (1.6) and (1.7) are consequences of identities (1.8) and (1.9).

\section{Acknowledgments}

The authors really appreciate the valuable suggestions given by referees for improving the overall quality of the manuscript, especially for pointing out the polynomiality of our Plancherel averages in $t$. The second author is supported by Grant [P2ZHP2_171879] of the Swiss National Science Foundation. He also thanks Prof. P.-O. Dehaye for the encouragements and helpful suggestions.

\section{REFERENCES}

[1] R. M. Adin and A. Frumkin, Rim hook tableaux and Kostant's $\eta$-function coefficients, Adv. Appl. Math. 33(3) (2004), 492-511.

[2] J. Bandlow, An elementary proof of the Hook formula, Electron. J. Combin. 15 (2008), research paper 45 .

[3] A. M. Borodin, Multiplicative central measures on the Schur graph, J. Math. Sci. 96 (1999), 3472-3477.

[4] P.-O. Dehaye, G.-N. Han, and H. Xiong, Difference operators for partitions under the Littlewood decomposition, Ramanujan J. 44(1) (2017), 197-225. 
[5] S. Fujii, H. Kanno, S. Moriyama, and S. Okada, Instanton calculus and chiral one-point functions in supersymmetric gauge theories, Adv. Theor. Math. Phys. 12(6) (2008), 1401-1428.

[6] F. Garvan, D. Kim, and D. Stanton, Cranks and t-cores, Invent. Math. 101 (1990), 1-18.

[7] G.-N. Han, The Nekrasov-Okounkov hook length formula: refinement, elementary proof, extension, and applications, Ann. Inst. Fourier 60(1) (2010), 1-29.

[8] G.-N. Han, Some conjectures and open problems on partition hook lengths, Experimental Mathematics 18 (2009), 97-106.

[9] G.-N. Han, Hook lengths and shifted parts of partitions, Ramanujan J. 23(1-3) (2010), 127135.

[10] G.-N. Han and K. Q. Ji, Combining hook length formulas and BG-ranks for partitions via the Littlewood decomposition, Trans. Amer. Math. Soc. 363 (2011), 1041-1060.

[11] G.-N. Han and H. Xiong, Difference operators for partitions and some applications, preprint; Ann. Combin., to appear.

[12] G.-N. Han and H. Xiong, New hook-content formulas for strict partitions, Algebraic Combin. 45(4) (2017), 1001-1019.

[13] V. N. Ivanov, Dimensions of skew-shifted Young diagrams and projective characters of the infinite symmetric group, J. Maht. Sci. 96 (1999), 3517-3530.

[14] G. James and A. Kerber, The Representation Theory of the Symmetric Group, Encyclopedia of Mathematics and its Applications, 16, Addison-Wesley Publishing, Reading, MA, 1981.

[15] D. Knuth, The Art of Computer Programming, Vol. 3: Sorting and Searching, AddisonWesley, London, 1973, pp. 54-58.

[16] A. Lascoux, Symmetric Functions and Combinatorial Operators on Polynomials, Vol. 99, American Mathematical Soc., 2003.

[17] I. G. Macdonald, Symmetric Functions and Hall Polynomials, Oxford Mathematical Monographs, The Clarendon Press, Oxford University Press, New York, second edition, 1995.

[18] S. Matsumoto, Polynomiality of shifted Plancherel averages and content evaluations, preprint; arXiv: $1512.04168 \mathrm{v} 3$.

[19] S. Matsumoto, Plancherel measures on strict partitions: Polynomiality and limit shape problems, Workshop on Asymptotic Representation Theory, February 20-24 2017, available at http : //combi17.math.cnrs.fr/Slides_ART/Matsumoto_ART.pdf.

[20] S. Matsumoto, Correlation functions of the shifted Schur measure, J. Math. Soc. Japan 57 (2005), 619-637.

[21] N. A. Nekrasov and A. Okounkov, Seiberg-Witten theory and random partitions, The unity of mathematics, Progress in Mathematics 244, Birkhäuser Boston, 2006, pp. 525-596.

[22] G. Olshanski, Anisotropic Young diagrams and infinite-dimensional diffusion processes with the Jack parameter, Int. Math. Res. Not. IMRN 6 (2010), 1102-1166.

[23] G. Olshanski, Plancherel averages: Remarks on a paper by Stanley, Electron. J. Combin. 17 (2010), research paper 43.

[24] G. Panova, Polynomiality of some hook-length statistics, Ramanujan J. 27(3) (2012), 349356.

[25] M. Pétréolle, A Nekrasov-Okounkov type formula for $\widetilde{C}$, Adv. Appl. Math. 79 (2016), 1-36.

[26] M. Pétréolle, Quelques développements combinatoires autour des groupes de Coxeter et des partitions d'entiers, Ph.D. thesis, 2015.

[27] G.-C. Rota, On the foundations of combinatorial theory: I. Theory of Möbius functions, Z Wahrscheinlichkeitstheorie und Verw. Gebiete 2 (1964), 349-356.

[28] I. Schur, Über die Darstellung der symmetrischen und der alternienden Gruppe durch gebrochene lineare Substitutionen, J. Reine Angew. Math. 139 (1911), 155-250.

[29] R. P. Stanley. Some combinatorial properties of hook lengths, contents, and parts of partitions, Ramanujan J. 23(1-3) (2010), 91-105.

[30] R. P. Stanley, Differential posets, J. Amer. Math. Soc. 1(4) (1988), 919-961.

[31] R. P. Stanley, Enumerative Combinatorics, vol. 2, Cambridge University Press, New York/Cambridge, 1999.

[32] R. M. Thrall, A combinatorial problem, Michigan Math. J. 1 (1952), 81-88.

[33] B. W. Westbury, Universal characters from the Macdonald identities, Adv. Math. 202(1) (2006), 50-63.

Université de Strasbourg, CNRS, IRMA UMR 7501, F-67000 Strasbourg, France

Email address: guoniu.han@unistra.fr, xiong@math.unistra.fr 